\title{
Automated Discovery of Big Data Workload Types
}

\author{
by
}

\author{
Anousheh Shahmirza \\ A thesis submitted to the \\ Faculty of Graduate and Postdoctoral Affairs \\ in partial fulfillment of the requirements for the degree of
}

\begin{abstract}
Master of Science
in

Computer Science with Specialization in Data Science
\end{abstract}

Ottawa-Carleton Institute of Computer Science

Department of Computer Science

Carleton University

Ottawa, Ontario

August, 2021

CCopyright

Anousheh Shahmirza, 2021 
The undersigned hereby recommends to the

Faculty of Graduate and Postdoctoral Affairs acceptance of the thesis

\title{
Automated Discovery of Big Data Workload Types
}

\author{
submitted by Anousheh Shahmirza \\ in partial fulfillment of the requirements for the degree of \\ Master of Science \\ in \\ Computer Science with Specialization in Data Science
}

Professor Amiya Nayak, Ph.D, External Examiner

Professor Frank Dehne, Ph.D, Thesis Supervisor

Dr. Michael Genkin, Ph.D, Thesis Co-supervisor

Professor Anil Somayaji, Ph.D

Ottawa-Carleton Institute of Computer Science

Department of Computer Science

Carleton University

August, 2021 


\section{Abstract}

Big data workload characterization is an inevitable part of big data workload prediction and auto-tuning big data applications. Due to many different ways of applying big data frameworks and applications, there are various categories that these workloads can belong. Methods such as classifications that employ historical records are not beneficial since they require humans to assemble training sets for these workloads in order to build a model, which is ultimately expensive. Consequently, it is necessary to classify these workload streams automatically and without human intervention. Clustering techniques are applied in this research to detect Apache Spark and Hadoop workloads independent of historical data. Clustering techniques are compared in terms of different evaluation metrics, and the ones with the highest performance are introduced. The DBSCAN algorithm has shown the best performance and adequacy with $71 \%$ and $80 \%$ for the Purity, and Windows Type Accuracy (Awt), respectively. Ultimately, the Incremental DBSCAN algorithm and Den-Stream (an online version of DBSCAN) are presented as the most practical methods for big data workload discovery automatization. A scheme is then provided to use these algorithms integrated with methods to self-discover their hyperparameters. Ultimately, the procedure is fully automated. The proposed prototype automatically discovers the Apache Hadoop and Spark workloads' data stream, with a high degree of accuracy, same as those identified by an expert human practitioner. 
This thesis is dedicated to my parents, Nadereh Fardyar and Reza Shahmirza. Thank you for your love and endless support, this endeavour would not have been possible without you. 


\section{Acknowledgments}

I would like to extend my special thanks and appreciation for the encouragement and the unwavering belief given to me by professor Frank Dehne, the Chancellor's Professor in the School of Computer Science at Carleton University. I am both honoured and fortunate to have been one of your students.

I would also like to express my special appreciation to Ph.D. Candidate Mikhail Genkin. Please accept my heartfelt gratitude for your time, unconditional support and for sharing your profound knowledge of Big Data. I have also been blessed to have the opportunity to work with a knowledgeable and proficient team of researchers.

I wish to thank Dr. Anil Maheshwari, my academic advisor, who helped me throughout this journey.

I would like to thank Professor Dr. Amiya Nayak, and Professor Dr Anil Somayaji for serving as my committee members. 


\section{Table of Contents}

Abstract

Acknowledgments $\quad$ v

Table of Contents $\quad$ vi

List of Tables $\quad$ ix

List of Figures $\quad$ x

1 Introduction 1

1.1 Motivation . . . . . . . . . . . . . . . . . . 1

1.2 Problem Statement . . . . . . . . . . . . . . . . . . . . . . . 1

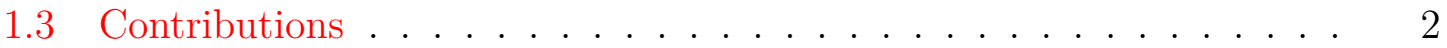

1.4 Thesis Outline $\ldots \ldots \ldots \ldots \ldots \ldots \ldots \ldots$

2 Background 4

2.1 Big Data and Parallel Computing . . . . . . . . . . . 4

2.1.1 MapReduce ...................... . 5

2.1.2 Review of Hadoop . . . . . . . . . . . . . . . . 6

2.1.3 Review of Spark . . . . . . . . . . . . . . . . 6

2.1.4 Spark and Hadoop Infrastructure . . . . . . . . . . 7 
3 Literature Review $\quad 10$

3.1 big data workload characterizations . . . . . . . . . . . . . . . 10

3.2 Clustering Algorithms . . . . . . . . . . . . . . . . . 18

3.2.1 Density Based Methods . . . . . . . . . . . . . . . 19

3.2.2 Partition Based Methods . . . . . . . . . . . . . . . . 21

3.2.3 Hierarchical Methods . . . . . . . . . . . . . . . . . . . 22

3.2.4 Find optimum number of clusters . . . . . . . . . . . . . . 22

3.2.5 Grid Based Methods . . . . . . . . . . . . . . . 23

3.2.6 Model Based Methods . . . . . . . . . . . . . . 23

4 Methodology $\quad 24$

4.1 Apache Spark and Hadoop Workload Identification . . . . . . . . . . 24

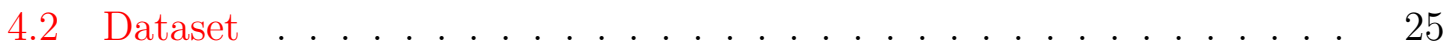

4.2.1 Wordcount ................... 27

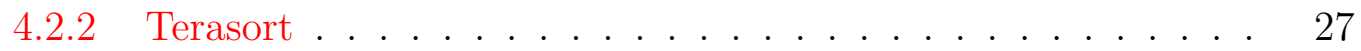

4.2.3 Terasort and HiBench K-means . . . . . . . . . . . . . . 28

4.3 Data Preprocessing . . . . . . . . . . . . . . . . . . 29

4.4 Evaluation Metrics . . . . . . . . . . . . . . . . . . 29

$\begin{array}{lll}5 & \text { Results } & 33\end{array}$

5.1 Experimental Implementation and Setup . . . . . . . . . . . . . 33

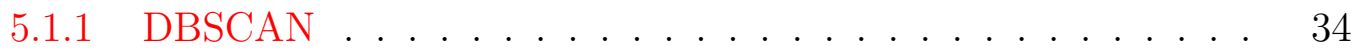

5.1 .2 Optimum number of clusters ............. 36

5.1.3 Mean Shift Clustering . . . . . . . . . . . . . 36

5.1.4 K-Means Clustering . . . . . . . . . . . . . . 37

5.1.5 Expectation Maximization (EM) clustering . . . . . . . . 37

5.1.6 Hierarchical Clustering . . . . . . . . . . . . . . . . . . . . 38 
5.2 Performance Analysis . . . . . . . . . . . . . . . . . . 38

5.3 Discussion . . . . . . . . . . . . . . . . . . . . . 42

6 Conclusions and Future Work 48

$\begin{array}{ll}\text { List of References } & 50\end{array}$ 


\section{List of Tables}

4.1 Observation window features. Table from [17] . . . . . . . . . 26

4.2 Workloads types. Table from [17]. . . . . . . . . . . . . . . 27

4.3 Workloads transition types. Table from [17] . . . . . . . . . . . 27

4.4 An example of the data set . . . . . . . . . . . . 28

4.5 An example of the ground truth set . . . . . . . . . . . . 29 


\section{List of Figures}

2.1 MapReduce Processing Scheme. . . . . . . . . . . . . . . . 5

5.1 Chart for the average DBSCAN accuracy for four different data files. 35

5.2 Chart for the DBSCAN accuracy for the data set Tera-Kmean. Kmeans starts running concurrently with Terasort when Terasort just started its reduce phase. . . . . . . . . . . . . . . . 36

5.3 Chart for the DBSCAN accuracy for the data set Hibench-Wordcount 37

5.4 Chart for the DBSCAN accuracy for the data set Terasort . . . . . 38

5.5 Chart for the DBSCAN accuracy for the data set Tera-K-means. Kmeans runs concurrently with Terasort. . . . . . . . . . . . . . . 39

5.6 Knee plot for Tera-K-means. K-means starts running concurrently with Terasort when Terasort just started its reduce phase. . . . . . . 40

5.7 Knee plot for Tera-K-means. K-means runs concurrently with Terasort. 41

5.8 Knee plot for Hibench-Wordcount. . . . . . . . . . . . . . . . 41

5.9 Knee plot for Terasort. . . . . . . . . . . . . . . . . . . . . 42

5.10 Chart for the comparison of the average accuracy in different clustering algorithms . . . . . . . . . . . . . . . . 4 43

5.11 Chart for the comparison of the metrics in different clustering algorithms for the Tera-K-means workloads. K-means starts running concurrently with Terasort when Terasort just started its reduce phase. . 
5.12 Chart for the comparison of the metrics in different clustering algorithms for the Hibench-Wordcount workloads . . . . . . . . . . . . . 45

5.13 Chart for the comparison of the metrics in different clustering algorithms for the Terasort workloads . . . . . . . . . . . . . . . . . 46

5.14 Chart for the comparison of the metrics in different clustering algorithms for the Tera-K-means workloads. K-means runs concurrently with Terasort. . . . . . . . . . . . . . . 


\section{Chapter 1}

\section{Introduction}

\subsection{Motivation}

Discovering big data workloads types gives users an insight into the required resources. Due to the limitations of the systems, it is essential to optimize resource usage. Many attempts have taken place to characterize big data workloads. None of these studies have worked on automated discovery of the workloads characterization. Moreover, most of the studies are based on system-level metrics. However, Genkin and Dehne [17] worked on higher-level metrics targeted in this research. In this study, the researchers are trying to improve automation proposed by Genkin and Dehne [17].

\subsection{Problem Statement}

Previous study [17] used the random forest to separate and identify the workloads streams, then anticipated future workloads. They demonstrated that it is possible to accurately classify big data workloads using supervised machine learning algorithms, such as the Random Forrest algorithm. These algorithms, however, are only able to classify labeled workloads that were present in the training set. If a brand new 
workload was executed, the algorithm would not be able to recognize it and would erroneously classify the new workload as one of the previously seen workloads. This is a problem. The main focus of this thesis is to use unsupervised machine learning algorithms to identify previously unseen big data workloads. Since no semantic label is required for the training set, instead of using a training set that humans have labelled, we characterize these workloads using clustering algorithms and automatically generate labels to assign them. Consequently, the training set required for the random forest phase used in [17] is regularly updated. The dataset is not restricted to labels in the training set, and new workloads can be recognized and appropriately characterized.

\subsection{Contributions}

This research work is built on previous efforts [17] done by my colleagues and supervisors Frank Dehne, and Mikhail Genkin. An improvement of the workload identifica-

tion section of the previous study, "Autonomic Workload Change Classification and Prediction for Big Data Workloads" [17] was the goal of this study. The contributions of this thesis:

- This thesis is the first study to evaluate the effectiveness of different clustering algorithms on big data workloads.

- This thesis has devised a method to automate the discovery of big data workload types. 


\subsection{Thesis Outline}

Chapter 2 provides a brief background of big data and parallel computing, especially Hadoop and Spark, which the researchers have been focused on. Chapter 3 is dedicated to previous research in the scope of big data work characterization as well as unsupervised learning methods. All the clustering algorithms that are used in the experiments are defined and explained in Chapter 3. A description of the research methodology is included in Chapter 4. The benchmarks that are used for the assessment, how the data was prepared for analysis, and the explanation of the different state of the art clustering performance metrics are also presented in this chapter. Chapter 5 presents an analysis of different clustering algorithms and compares the results. The two best clustering methods are detected for discussion in order to then conduct a strategy for the application of the online version of each for automation pur-

poses. In chapter 6 , the researchers illustrate a summary of the proposed prototype, and suggests potential future work that may improve the results of this study. 


\section{Chapter 2}

\section{Background}

\subsection{Big Data and Parallel Computing}

Analyzing, storing, and extracting massive datasets requires advanced analytic techniques. Decision-making in most fields, from civil engineering to biotechnology and social network analysis, is based on analyzing the historical data. Due to these essentials and the challenges, the concept of big data has arisen. Big data provides data mining and machine learning methods in order to make use of as much data as possible.

Parallel computing is a computing architecture in which multiple executors are applied synchronously to execute a computation. Process workloads are divided between the executors to run at the same time. By distributing the computations across hundreds or thousands of machines, the execution time is reduced to a reasonable amount of time. In this architecture, a set of networked computers is called a cluster. Each computer is named a node of that cluster. The memory in parallel processing could be shared among all the cluster nodes, distributed in which each node has its memory or a combination of both. 


\subsubsection{MapReduce}

MapReduce framework has been devised to deal with big data in parallel. With MapReduce, massive data is processed by connecting many cluster nodes to work in parallel. The data is split into smaller chunks. These chunks are then processed concurrently, and the results of all servers are aggregated to return the output. To speed up the procedure rather than sending the data where the application is located, MapReduce sends the logic where the data is located [41].

Both the Map function and the Reduce function are supposed to be programmed. They take a key-value pair as input and may output key-value pairs. This algorithm starts with applying a map operation to each logical record in the input data to compute a set of intermediate pairs in the format of $<$ key, value $>$. Then, it employs a Reduce operation to all the values that share the same key in order to combine the derived data appropriately [10]. Moreover, there could be optional processes between the Map and Reduce functions. The input and output of all these processes are key-value pairs.

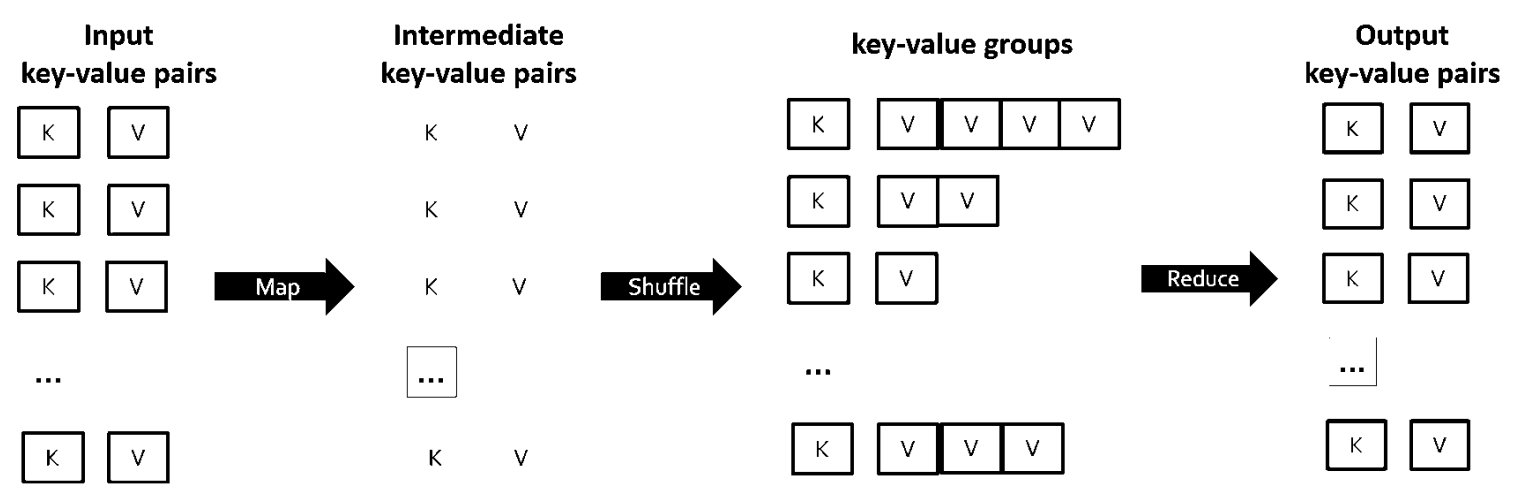

Figure 2.1: MapReduce Processing Scheme. 


\subsubsection{Review of Hadoop}

Google's MapReduce, called Hadoop, is a practical framework for executing the applications in parallel. One major problem dealing with massive datasets is data storage. It takes too long to read data from a single drive. In this architecture, the data is divided between multiple clusters. Hadoop reads the data from multiple disks at once and processes them in parallel. In many situations, the data from one disk needs to be aggregated with the data from another disk. Using the MapReduce framework, Hadoop is able to deal with combining the data from different disks [45].

The idea behind the Hadoop Distributed File System (HDFS) is the efficiency of the write-once and read-many-times patterns. HDFS follows a master-worker pattern. It has a NameNode (the master) and one or many DataNodes (workers). The Master node is responsible for controls the system in terms of resource management, job scheduling, job tracking, and failure. Data is distributed across the DataNodes, where the logic resides [45].

\subsubsection{Review of Spark}

Apache Spark is an open-sourced programming model that supports a much broader class of applications and operations than MapReduce. Apache Spark has excellent performance for multipass applications that require low-latency data sharing across multiple parallel operations. In light of query optimization and in-memory caching, Apache Spark is much faster than Hadoop [42]. Resilient Distributed Dataset (RDD) is the data structure used in Apache Spark that can be executed in parallel by processors of the clusters. After applying any RDD operation, a new RDD is created, and the original one remains the same. Consequently, there is always the option to go back in case of failure [42]. 
Spark executes a function that is passed by the user to run on each executer. This function belongs to a serializable object and needs to be serialized before sending it to the cluster nodes. As a result, each worker has a copy of the variables used in the function that belongs to them [52].

Spark supports the abstraction of shared variables. In many cases, a variable needs to be shared among workers or workers and the driver. Two types of shared variables exist: Broadcast variables, which are read-only, and each executor has a local copy of these variables as well as their values. Accumulator variables that can be updated by the executors using their "add" function. However, only the driver can read these variables. These variables are mostly used as counters in parallel sums [52].

\subsubsection{Spark and Hadoop Infrastructure}

The huge hardware clusters for big data engines such as Hadoop, Spark, Hive, and Hbase have a Resource Manager on the master node, which communicates with the Node Manager of each data node to trace their tasks and allocate resources on each data node. Moreover, the Resource Manager is in charge of deciding about the usage of CPU, memory, and disk on the cluster. Some of the existing resource managers are YARN, Mesos, and Kubernetes. [18].

The YARN Resource Manager has been targeted in this study. The reason for studying YARN in this research is its capability to be used by both the Hadoop and the Spark frameworks. As a result, the researchers did not need to produce the data in two different ways for each of the Hadoop and Spark. In this research, the container performance metrics are the same for all resource managers. As a result, the study's

contributions are not limited to YARN and could be used by other resource managers as well. 
YARN, which stands for Yet Another Resource Negotiator, is a Resource Manager [3] containing two major modules: Scheduler and Applications Manager. The scheduler is in charge of deciding about resource allocation based on the application requirements and the capacity of the memory, CPU, Disk, network, etc. The Application Manager is in charge of sending the start command, handling failures, controlling, reporting and managing the progress. The Resource Manager communicates with a fraction of Node Managers in each data node called containers for this purpose. The YARN architecture is shown in [13].

A big data routine starts when a client submits a job to the Resource Manager. Then, the resource manager allocates a container on each Node Manager of the Data nodes. After that, the Resource Manager commands the Node Managers to begin the process. The Node Managers then initiate the container. In the end, the containers execute the application masters. The Node Manager is responsible for sending a report to the Resource Manager regularly.

Before continuing, it is essential to define some of the frequently used terms for this study [17]:

- Observation Window: The container reports its condition based on specific time intervals called the observation window, which is a real-time stream. The Observation Window feature vector contains real numbers. Table 4.1 describes each of the features.

- Workload: A series of Observation Windows with almost the same characteristics illustrates a constant procedure called a workload. For instance, in the Hadoop concept, any continuous map, reduce, and shuffle operations are considered a workload. In the Spark context, each job is divided into different stages. Each of these stages is considered as a workload. Different workload 
types are illustrated in the 4.2 .

- Workload Transition: The switch from a workload to another workload is called Workload Transition. Workload Transitions are detected by observing a remarkable difference between the two consecutive observation windows' feature vectors. The Workload Transition contains one or many observation windows between two series of steady-state observation windows related to workloadsfor instance, changing the workload from the Shuffle phase to Reduce in the Hadoop map-reduce procedure. Table 4.2 explains different kinds of Workload Transition types.

However, in the case of running multiple Spark or Hadoop applications concurrently, the transitions would not be as clear as a single application's execution. For instance, the transition to the shuffle phase could be followed by another shift to the shuffle phase. 


\section{Chapter 3}

\section{Literature Review}

In section 3.1, previous methodologies and studies to characterize big data workloads are described. Section 3.2 contains the definition of the clustering algorithms that are considered in this research.

\section{1 big data workload characterizations}

Big data workload characterization studies have put effort into classifying big data workloads using different feature vectors. The research studies [33] and [34] target the operating system-level metrics. To date, [18] is the only research that puts effort into classifying big data workloads using the container performance patterns. The research themes of other studies, such as [27], [51], [5], and [46], focus on either microarchitectural-level characteristics or job-level characteristics.

- Microarchitectural-level metrics are characteristics such as instruction per cycle, L1 cache miss ratio, L2 cache miss ratio, last level cache miss ratio, number of branches per instruction, branch miss prediction per instruction, execute time breakdown, and off-chip bandwidth [5].

- Job-level metrics contains data processing speed, the ratio of the map output 
concerning the input, the ratio of the shuffle stage to the respective map, the ratio of the map phase duration to the reduce phase duration, the rate of the reduce output data to the reduce input data, and the ratio of the reduce output data to the map input data [51].

- System-level characteristics are metrics such as CPU and memory usage, bandwidth, and disk utilization capacity.

- Container performance metrics includes container creation rate, average response-time of containers started, relative standard deviation of containers started, the rate of completed containers, average response-time of containers finished, the relative standard deviation of containers finished, average actual CPU utilization of the cluster, average actual active memory utilization on the cluster, average genuine network read rate on the cluster, the mean of actual network write rate on the cluster [17].

Most of the references reviewed in this study analyze the single-user workloads. However, [18] is dedicated to multi-user workloads. The real-life big data workloads emerge from multiple applications running concurrently.

One of the early attempts at characterizing big data workloads [33] devises a methodology for the workloads classification with respect to the resource consumption characteristics. It characterizes the Google Cloud backend workloads [23] used for scheduling purposes. They discover CPU and memory usage as the critical features for scheduling. Consequently, in terms of the feature vector, they consider the CPU usage in cores, memory usage in gigabytes, and each workload execution duration in seconds. First, the workload clusters are generated using standard clustering techniques such as k-mean. Then, the boundaries of quantitative features are identified. These boundaries are then used to convert the data into a qualitative data set. 
A label is going to assign to each cluster according to their qualitative coordinates. Finally, the adjacent classes are merged based on these labels to reduce the number of workloads. In order to convert the quantitative features to qualitative, the duration of each workload is transformed as either small (short) or large (long). The CPU consumption and memory consumption attributes are converted to small, medium, or large. These boundaries are chosen manually to create a low coefficient of variation (CV) for each feature. $\mathrm{CV}$ is computed as the fraction of the standard deviation to the mean [1]. The members' mean value for each qualitative attribute is calculated for each cluster. Then, these values are converted to qualitative values. The clusters are labeled according to these three values. For example, consider a cluster with the value 'small' as time, the value 'large' as CPU usage, and the value 'small' as memory usage. The label for this cluster would be SLS. Therefore, there are 18 different classes. Finally, these adjacent classes are merged to reduce the number of different classes. The adjacent classes are merged if the merged class's CV is almost the same as the CV for each of the involved classes. For instance, the classes LLM, LMM, and LSM have the potential to be merged as $\mathrm{L}^{*} \mathrm{M}$.

Xiong et al. [46] focus solely on the characterization of Hadoop workloads. They employed HiBench [25] and ICTBench [32] benchmarks to generate the workloads for their research. The microarchitectural attributes that are targeted by the researchers in this study are: branch miss prediction per instruction, the maximum number of synchronize instruction in one cycle, execute time breakdown and off-chip bandwidth, L1 instruction cache miss ratio, L2 instruction cache miss ratio, as well as last level cache miss ratio. Their setup consists of a master and four slaves. Each metric-node pair is considered as a variable in their design. Consequently, characterizing is based on 28 properties. The data is first normalized to have all the properties in the same range. Then the PCA [28] is applied to create a new set of uncorrelated attributes. 
Finally, Single Linkage Hierarchical clustering [48] with Euclidean distance is used to distinguish the different workloads.

Moreno and colleagues worked on characterizing the Cloud user-driven workloads. Their proposed method is applied to Google traces [22], which is one of the most largescale data centers. [34] considers the relationship between the actors and the tasks in the Cloud environment. An actor is in charge of managing the volume of tasks in the cloud system. The dataset features consist of the trace span, number of tasks, number of users, average task length, number of servers, average tasks per day, average users per day, and average tasks per user. Moreno and colleagues employ the K-means clustering algorithm to group the data into $\mathrm{K}$ different partitions. $\mathrm{K}$ is determined based on the strategy introduced by Pham et al. [36].

Jia et al. study the effects of software stacks such as Hadoop and Spark on big data workload using ultra low-level performance characteristics [27]. Thirty-two BigDataBench's workloads [44] and a feature vector of the length forty-five for each workload are given. PCA [28] is used to identify the most critical workload features. Kaiser's Criterion is used to choose the number of principal components (PCs). Consequently, only those best PCs with eigenvalues greater than or equal to one are considered. According to Kaiser's Criterion, the resulting data would be sufficiently representative of the initial data. Then, Single Linkage Hierarchical clustering [48] is applied to find similar big data workloads and subset big data workloads. The Euclidean distance is chosen as a distance measure in this study.

Awan et al. distinguished the Batch and Stream Workloads in Apache Spark applications [5]. They also compare the performance between DataFrames and RDDs in Spark applications. Micro-architectural Characterization is targeted in this research. The workloads are produced by three big data benchmark suits which are BigDataBench [44], HiBench [25], and StreamBench [31]. They put in practice the 
top-down approach introduced by Yasin [50]. Four pipeline slots per clock cycle for each CPU core are considered to classify the micro-operations. The four slots are labeled as Front-end Bound, Back-end Bound, Bad Speculation and Retiring. Regarding the micro-batching differences, comparing the flagged domains illustrates that the micro-architectural level attributes on batch and stream processing show the same pattern. Moreover, their research asserts that spark workloads using DataFrames performs better than spark workloads using RDDs. They state that since a DataFrame is a data structure with rows of 8 bytes aligned, the hashing and search operations perform better than the RDDs, which uses Java objects.

A recent study [51] with a concentration on Hadoop has been done. However, the authors argue that their methodology can be applied to other big data frameworks such as Spark as well. They worked on the SZTS [47], HiBench [25], and CloudRank-D [32]. They consider both the microarchitecture-level and job-level metrics as Hadoop workloads characteristics. Consequently, the characterization is dealing with too many attributes. Their study [51] devises a strategy called Metric Importance Analysis to rank each metric based on its relative importance. A Kiviat plot [6], and a Similarity Matrix based on these ranked metrics is provided for each workload to illustrate the similarity and dissimilarity among the benchmarks. The stochastic gradient boosted regression (SGBR) [15] is employed for the metric importance analysis. The SGBR uses an ensemble of regression tree algorithms to predict the importance of each attribute. $n$ most important metrics are chosen as the Kiviat plot axis in descending order in a clockwise direction. Yu et al. claim the $n$ is the minimum number of metrics in which the sum of the importance surpasses 80 percent of the sum of all metrics importance. Each feature in the dataset is normalized using the Min-Max method 4.1 to generate comparable Kiviat plots. Considering a similarity benchmark matrix, a weighted feature value vector for each benchmark is 
created. They then calculate the similarity and dissimilarity among the benchmarks using Manhattan Distance. As it is mentioned in section 2.1.4, the Hadoop and Spark Resource Manager use a construct of the worker nodes called Container. The Resource Manager has many configurable parameters that affect the performance of the Apache Hadoop and Spark. In order to choose the optimal parameters manually, the analytic job is supposed to be executed with all the combinations of the parameters. Then, the parameters with the best performance are chosen. Big data applications take hours to days to be executed. As a result, manual tuning of the parameters does not seem to be reasonable.

Recently, a study [18] records achievements regarding the container performance metrics, the key points to online auto-tuning systems. They analyze the log files resulting from executing a Hadoop or Spark job. The observation windows' duration is chosen based on having a statistically sufficient number of container records on most of the observation windows. Consequently, a couple of Spark and Hadoop jobs are executed to find each time interval's optimum duration. Their research study reveals the uniqueness of the container routines. It classifies the workloads as either Hadoop or Spark based on container properties. Moreover, it introduces a method to detect workload changes. Performance metrics have been introduced for the Hadoop and Spark workloads. These metrics are defined per each observation window. These metrics are the number of containers created, the number of containers that complete the execution, the average response time for all containers that are done the execution, and the standard deviation of container response-times divided by the mean of container response-time. These metrics are calculated for each observation window, as well as the relative amount of changes that occur after each transaction between workloads. The relative amount of changes is calculated as the mean of these metrics for the two viewer window after the transition, divided by this mean just before the 
transition. Genkin and Dehne state that these metrics' values are at least three times more for Hadoop than Spark. Their study illustrates that the Random Forest classifier performs the workload classification with 83 percent accuracy. Using feature selection techniques, they figure out that the container creation attribute is the only relevant feature that results in the best accuracy.

In terms of change detection, observation window data is considered as a real-time stream. The moving average and the standard deviation of each of the container metrics are calculated for the five windows in a row. Then, Welch's test is applied to validate the difference between the means calculated between every two consecutive windows. Double-sided Welch's test is considered with the confidence of 95 percent. A transition has occurred if Welch's test result is valid. For transition detection, the container creation rate feature demonstrates the least accuracy. However, the relative standard deviation of response-time (RSD) produces the most accurate detection with an accuracy of 74 percent.

Big data frameworks, such as Apache Spark and Hadoop, are built upon many configurations and settings, which are chosen either manually or automatically. The study [17] which is the basis of this research, is focused on auto-tuning big data stack by predicting the workloads. In order to preserve the performance in terms of time and resources, it is required to re-tune the system only when there are any changes in the profiles of workloads. As a result, it is required to predict the future workload and detect the changes in the features. Moreover, this becomes more challenging when it comes to running multiple jobs synchronously. To predict the changes in a workload, analyzing the workload attributes in the past and detecting special situations would not be useful. Consequently, the aim is to predict the near workload type, which is not a result of analyzing the historical data. The reason is that big data engines do not follow any specific patterns. Especially in the case of transitions in running 
concurrency jobs, the workloads' characteristics change from CPU to Disc or vice versa. These alterations may cause other workload changes, such as discarding the start or finish of a job.

Studying the observation windows produced by the resource manager, Genkin and Dehne [17] were able to anticipate bigdata workloads. The prediction architecture contains four main levels described as follows:

1. ChangeDetector: In this phase, observation windows are classified as a steadystate workload or a workload transition using an unsupervised and online method. The Welch's [21] test is applied to each feature vector's element. The $\mathrm{N}$ last observation windows are used to calculate the mean and the standard deviation for each observation window feature.

$$
t=\frac{\overline{X_{1}}-\overline{X_{2}}}{\sqrt{\frac{\sigma_{1}^{2}}{N_{1}}+\frac{\sigma_{2}{ }^{2}}{N_{2}}}}
$$

If a significant difference is detected based on Welch's test for a feature, one vote is added up. There could be some weights for each of the features as well. The ChangeDetectorvotes aggregates the votes. If it is greater than a predefined threshold, the observation window is detected as a transition workload. The optimum threshold and the weights are obtained after applying multiple experiments off-line. The ChangeDetector performs with $99 \%$ accuracy.

2. WorkloadClassifier: The observation windows detected as steady-state workload by the ChangeDector, are classified in this phase. A combination of random forest classification [30] is trained off-line to detect the type of the current workload. Workload types are illustrated in table 4.2. The WorkloadClassifier demonstrated $90 \%$ accuracy. 
3. TransitionClassfier: The observation windows detected as a transition workload by the ChangeDector, are classified in this stage. This classifier uses the same combination of classifiers as the WorkloadClassifier. However, it does not analyze the observation window. Instead, this classifier's input parameter is the rate of changes of the current window feature vector compared with $\mathrm{N}$ last windows. The number of windows to participate in the rate calculation is chosen based on the experience. Similar to the WorkloadClassification, the TransitionClassifier achieved 90\% accuracy.

4. WorkloadPredictor: The previous two classifications produce a series of labels assigned to each observation window like "HDMAP HDMAP HDMAP HDMAP HDMAP HDMAPSHUF HDMAPSHUF HDSHUF HDSHUF HDSHUF HDSHUFRED" [17]. The WorkloadPredictor applies a single layer LSTM(Long Short-Term Memory) [24] to predict the upcoming observation windows. LSTM is configured in this research to anticipate the ten upcoming workload or workload transition observation windows. The accuracy of the WorkloadPredictor was found to be approximately $96 \%$ accurate.

\subsection{Clustering Algorithms}

Unsupervised learning is a machine learning area of study in which the data does not have any label to learn from, or there is no feedback to give a hint of whether the learning is on the right track. Unsupervised learning aims to find patterns in the data and detect those which do not follow any patterns, these are called noise. Clustering techniques are a sub-field of unsupervised learning [20]. Clustering algorithms group data into a certain number of clusters so that the members of a cluster are as similar as possible while the members of two different clusters expose the dissimilarity as 
much as possible. Clustering methods are categorized as Partition based, Hierarchical based, Density-based, and Grid-based, as well as Model-based algorithms.

\subsubsection{Density Based Methods}

Density-based clustering algorithms are useful to find clusters of arbitrary shapes. DBSCAN, OPTICS, DBCLASD, GDBSCAN, DENCLU, and SUBCLU are classified as density-based clustering algorithms [38]. One of the most well-known Densitybased algorithms is DBSCAN [12], which is an acronym for Density-Based Spatial Clustering of Applications with Noise. DBSCAN discovers clusters of arbitrary shape and size and is resistant to noise. DSBSCAN has two hyperparameters:

- Eps. The maximum radius of the neighbourhood

- MinPts. The minimum number of points exist in an Epsilon-neighbourhood of a point

The DBSCAN procedure is as follows [12]:

1. Given a radius eps as well as the required number of points in a cluster minPts, as input parameters.

2. An arbitrary point $P$ is chosen.

3. All points in the eps neighbourhood of the $\mathrm{P}$ are retrieved.

4. If the number of P's neighbours is less than minPts, then $\mathrm{P}$ is flagged as a noise point, which could be converted to a cluster point in the future.

5. Else if the number of P's neighbours is enough to take shape a cluster, then this point is flagged as visited. $\mathrm{P}$ and all points in the eps neighbourhood of the $\mathrm{P}$ join the cluster. 
6. Steps 3 to 5 repeats for all the cluster points until all points in the cluster are flagged as visited.

7. Another unvisited point $P$ is chosen.

8. Steps 3 to 6 are applied to take shape a new cluster or noise.

9. The cycle keeps going until no unvisited point could be found.

One of the algorithms that could be used as an online version of DBSCAN is DenStream. Den-Stream stands for Density-Based Clustering over an Evolving Data Stream with Noise [7]. Den-Streams applies DBSCAN to the First InitN point to create p-micro-clusters. Then, for each new point of the stream, this algorithm attempts to join the point with one of the existing p-micro-clusters. If there are no clusters to join, then the DEN-STREAM makes an effort to merge that point with one of the outliers' micro-clusters and create an o-micro-cluster. DEN-STREAM defines a weight for each p-micro-clusters and o-micro-clusters. This weight reduces over time and increases after a new member is joined. In case the weight of an outlier micro-cluster exceeds the minimum weight, a cluster will take shape. In specific time intervals, the o-micro-clusters which does not meet the required weight to convert to a cluster are trimmed. Furthermore, a p-micro-cluster is removed if the weight of a p-micro-clusters has reduced to less than its required minimum weight. Accordingly, o-micro-cluster has the potential to convert to a p-micro-cluster.

DEN-STREAM has 5 hyper-parameters: Init $N, \epsilon, \beta, \mu, \lambda$. $\epsilon$ and $\mu$ are the DBSCAN parameters for the eps(radius) and minPts(minimumnumberofpoints), respectively. Init $N$ is the number of required initial points to apply a batch DBSCAN. $0<\lambda<1$ demonstrates the importance of historical data. $\beta$ is the outlier threshold. More outliers are found by increasing the $\beta$.

Aside from the Den-Stream, which is an online variant of the DBSCAN algorithm, 
and is used when there is a massive amount of stream data, there is the incremental DBSCAN algorithm [11]. Den-Stream keeps the center of the clusters and does not store the data points feature vectors. However, incremental DBSCAN preserves the data points feature vectors and adds the points one by one. [11] argues that only points in the specific neighbourhood of an inserted point are affected after insertion. Suppose Seed is the set of affected points after inserting a new point $\mathrm{p}$, incremental DBSCAN describes the different situations: (1) seed is empty; consequently, p is a noise point; (2) the Seed contains the point in which they were noise points before arriving p, but now a new cluster containing Seed and p takes shape; (3) the Seed contains points that belong to a single cluster; as a result, $\mathrm{p}$ joins to that cluster as well; (4) the Seed contains points that belong to different clusters, therefore, all the clusters' points along with the $\mathrm{p}$ join as one cluster.

\subsubsection{Partition Based Methods}

Partition based methods start with initial partitions and iteratively allocate point to different partitions to meet an optimum condition. Clustering algorithms such as K-means, K-medoids, K-modes, PAM, CLARA, CLARANS, and FCM are among the partition-based techniques [38].

Given the input parameter $\mathrm{K}$ as the number of clusters, K-means [48] starts with K randomly initialized points called clusters centroids. Then, each data point is assigned to its nearest cluster. The clusters' centroids are then updated by calculating the average of all the nodes in a cluster. This process iteratively continues until there is no change in the clusters [48]. As expected, K-medoids and K-modes follow the same algorithm as K-means. The difference is that instead of considering the mean of the points, the medroid (The point with the least average distance with other points of a cluster) of the points and the mode of points are computed, respectively. 
The Online format of Lloyd's algorithm [29] for K-means does not keep the data points in memory. The algorithm requires the number of clusters as an input parameter. It then, initializes $z_{1}, \ldots, z_{k}$ as cluster centers and keep the cluster sizes in variables $n_{1}, \ldots, n_{k}$. For each new data, the nearest cluster center $z_{i}$ is detected. The cluster size for the target cluster increased by one, and the cluster center is updated as $z_{i}<-z_{i}+1 / n_{i}\left(x-z_{i}\right)$.

\subsubsection{Hierarchical Methods}

Clustering algorithms such as BIRCH, CURE, ROCK, Chamelon, Echidna, Wards, SNN, CACTUS, and GRIDCLUST are categorized as Hierarchical clustering [38]. Hierarchical clustering starts with the most near two points and merges them. It then, calculates distances between clusters. The process stops after finding a predefined number of clusters or based on a density or radius threshold [48]. The results of this algorithm are highly dependent on how the distance between clusters is defined. Single Linkage is a method to define cluster distance, which is the distance between the two clusters' closest elements. Complete Linkage is another method for calculating the distance between clusters. It considers the distance between the farthest elements in two clusters. The distance between clusters in the Average Linkage is the average of all pair-wise distances between two clusters. The centroid method, however, calculates the distance between centroids.

\subsubsection{Find optimum number of clusters}

Many methods and techniques have been introduced to find the optimum number of parameters for the clustering algorithms that require the number of clusters as an input parameter. The NbClust package, which has been provided in $\mathrm{R}$ [8] executes 
30 different methods concurrently on the dataset. Each method is specified to find the best number of clusters. The optimum number of clusters is the number reported by the majority of the algorithms.

\subsubsection{Grid Based Methods}

Grid-based algorithms are STING, Wave cluster, BANG, CLIQUE, OptiGrid, MAFIA, ENCLUS, PROCLUS, ORCLUS, and FC STIRR [38]. Each feature of the data set is considered as a dimension of the space, and grids are taken shape. Dense regions of the grid are reported as clusters using subspace and hierarchical clustering algorithms. Grid-based clustering methods may produce overlapping clusters.

\subsubsection{Model Based Methods}

Methods such as EM, COBWEB, CLASSIT, and SOM, as well as SLINK, are considered as Model-based clustering algorithms [38].

EM algorithm, which is an acronym for the Expectation-Maximization clustering, is one of the renowned model-based clustering methods. EM clustering is a learning algorithm used to estimate the probability distributions' parameters best to fit the density of a given training dataset. It starts with $\mathrm{k}$ randomly distributions (Gaussians, e.g.), then for each point, it calculates the probability for that point belonging to each of the distributions. As a result, for each point, the cluster with the highest probability is chosen. Then the process continues until there is no change in the clusters [49]. The EM (expectation-maximization) technique is a generalized form of the k-means algorithm, with the assumption that the clusters are spherical, k-means results would be obtained. 


\section{Chapter 4}

\section{Methodology}

\subsection{Apache Spark and Hadoop Workload Identifi- cation}

This study is complementary to the classification pipeline [17] described in section 3.1. The Random Forest algorithm used to implement the WorkloadClassifier is a supervised learning algorithm that needs to be trained on labeled examples of workloads.

Clustering techniques used in this work enables the researchers to group the workload observation windows into different workload types. The reason for employing clustering methods was that clustering techniques are unsupervised techniques and do not need to be trained off-line. They can be used to identify the different workload types. This information can then be used to facilitate the training process for the supervised machine learning algorithms.

A prototype is designed to fully automate big data workload identification. Different clustering methods are compared and the two best methods are chosen. This study compares the offline clustering algorithms and analyzes the performance of different clustering methods on datasets containing a sufficient amount of received 
observation window as a result of executing Apache Spark or Hadoop applications. However, real-life big data workloads are received as data streams, and we need to assign each observation window to a proper cluster right after arrival. So the researchers studied the online version of the candidate algorithms and the capability to atomized them. Finally, the proposed techniques automatically choose the hyper-parameters for these algorithms and automate the identification process.

The researchers apply techniques to automatically choose the best parameters for these selected clustering methods for each of them. K-means clustering, Simple Hierarchical clustering, DBSCAN, Mean-shift clustering, and EM clustering are the focus of this study. Grid-based algorithms were not targeted in this experiment due to the possibility of producing overlapping results.

To obtain a meaningful comparison of different clustering algorithms' performance, it is required to figure out the algorithms' hyperparameter's optimum value. Then execute each algorithm with its optimum values for hyperparameters, and compare the results. The EM clustering, Hierarchical clustering, and K-means algorithm use the number of clusters as an input parameter. However, Meanshift clustering does not need any hyperparameter, as well as DBSCAN requires radius and the value for the minimum number of points in each cluster. The comparison of performance metrics discussed further in the Evaluation Metrics section of this chapter was then constructed.

\subsection{Dataset}

Several common Hadoop and spark time-series benchmarks are the targets in this study. This data sequence consists of big data jobs, which changes in terms of the type and number of workloads. These benchmarks were used as either a single-user or 
multi-user to analyze the clustering algorithms' performance on big data workloads. The reason for putting effort into analyzing the multi-user workloads is that big data jobs are usually executed synchronously in the real world. Each data file contains observation windows with features described in the table 4.1. Moreover, the information regarding the labels of each observation window was kept in a separate file. These ground truth sets of labels are the category of each window as well as the workload type in each observation window. The workload category is the category related to either a workload or a workload transition. The workload types are described in the tables 4.2 and 4.3. Tables 4.4 and 4.5 demonstrate samples of a dataset and a ground truth set.

KERMIT [19] logs were used to extract container properties. Ground truth files were prepared by analysing the Hadoop log files.

Table 4.1: Observation window features. Table from [17].

\begin{tabular}{ll}
\hline Feature Name & Description \\
\hline \hline numContStarted & Number of containers created during this observation window \\
meanStarted & Average response-time of containers started in this observation window \\
rsdStarted & Relative standard deviation of containers started in this observation window \\
numContainersFinished & Number of containers completed during this observation window \\
meanFinished & Average response-time of containers finished in this observation window \\
rsdFinished & Relative standard deviation of containers finished in this observation window \\
avgCPU & Average actual CPU utilization of the cluster during this observation window \\
averageActiveMem & Average actual active memory utilization on the cluster during this observation window \\
avgNetRead & Average actual network read rate on the cluster during this observation window \\
avgNetWrite & Average actual network write rate on the cluster during this observation window \\
\hline
\end{tabular}


Table 4.2: Workloads types. Table from [17].

\begin{tabular}{ll}
\hline Type & Description \\
\hline \hline HDMAP & Hadoop MapReduce Map phase processing \\
HDSHUF & Hadoop MapReduce shuffle processing \\
HDRED & Hadoop MapReduce Reduce phase processing \\
SPSTAGE & Spark job stage processing \\
SPSHUF & Spark shuffle processing \\
\hline
\end{tabular}

Table 4.3: Workloads transition types. Table from [17].

\begin{tabular}{ll}
\hline Type & Description \\
\hline \hline HDJSTART & Start of a Hadoop job. Could be under single or multi-user conditions \\
HDMAPSHUFL & Map ends and reduce-shuffle starts \\
HDSHUFRED & Reduce-shuffle ends \\
HDJEND & Reduce ends, end of Hadoop MapReduce job. Could be under single or multiuser conditions \\
SPJSTART & Spark job start \\
SPSTSTART & Transition from shuffle to the start of a new job stage \\
SPSTSTEND & Transition from a job stage to shuffle \\
SPJEND & Spark job end \\
\hline
\end{tabular}

\subsubsection{Wordcount}

The word count is one of the time series Hadoop map/reduce single-user benchmarking applications. It takes a collection of documents as input. The map phase assigns each word to a key-value pair <word, $1>$, it then sums over the value and returns the occurrences of each word as results in key-value format.

\subsubsection{Terasort}

Terasort (TeraByte Sort) [35] is a Hadoop single-user benchmark used to sort the data. Yahoo introduces Terasort. To execute the Terasort, the data is generated using the Teragen application. The Teragen creates a requested size data of random numbers compatible to use as Terasort input. Then, this data is sorted using the Terasort map/reduce algorithm. If there are N executers, Terasort keeps N-1 sample keys from data in a sorted array. The $<$ key, value $>$ data with the keys between 
sample(i-1) and sample(i) are assigned to the $i^{\text {th }}$ reducer. These sample keys are used to ensure that the data in the $i^{\text {th }}$ reducer is less than the data in the $(i+1)^{t h}$ reducer.

After the Terasort sorts the data, the TeraValidate key/value application is applied to confirm that it is sorted globally. The map phase of the Teravalidate returns the first and last keys of each output file. The reducer compares the last key of the $i^{\text {th }}$ file with the first key of the file $(i+1)^{t h}$. If the first key of the $(i+1)^{t h}$ file is not greater than the last key of the $i^{\text {th }}$ file, the reducer returns the unordered key.

\subsubsection{Terasort and HiBench K-means}

Terasort-K-means is a Hadoop multi-user benchmark, which means that multiple benchmarks are run synchronously. To create more interesting workloads that are more similar to the workloads produced by real-world applications, the researchers try to create a situation where the map phase of one application runs with the other application's reduce or shuffle phase, for instance, it starts by performing the Terasort benchmark, after the map phase of the Terasort is done, the K-means application is executed in parallel with the Terasort. The K-means benchmark is one of the benchmarks included in HiBench [25], which Intel introduced, it begins by generating random data points using statistical algorithms, the data points are clustered and labeled based on the k-means clustering algorithm.

Table 4.4: An example of the data set

\begin{tabular}{|c|c|c|c|c|c|c|c|c|c|c|c|c|c|c|c|c|c|c|}
\hline indowiD & numContainersStarted & meanStarted & 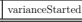 & statDerStarted & rsalStarted & \begin{tabular}{|l|} 
maxSStarted \\
\end{tabular} & minstarted & ImumContainersFinished & $\begin{array}{l}1 \text { mean Finished } \\
\text {. }\end{array}$ & varianceFinished & | stadervininished & \begin{tabular}{|l|l|} 
rralfinished \\
\end{tabular} & \begin{tabular}{|l|} 
maxFinishhed \\
\end{tabular} & 1 minFinished & avg с сри & avg active mem & a avg net read | & 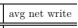 \\
\hline 0 & 178 & $\begin{array}{l}3.4369603 \\
\end{array}$ & 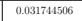 & 0.178169879 & 5.18393765 & 7.369066667 & 0.0 .6583 & 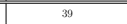 & 0.743109829 & $\begin{array}{ll}1.76 \mathrm{E}-\mathrm{M} \\
\end{array}$ & 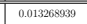 & 1.78555595916 & (0.98765 & 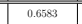 & $\begin{array}{l}67.9954762 \\
\end{array}$ & $\begin{array}{l}58.40 .601833 \\
\end{array}$ & 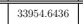 & 16278.88979 \\
\hline & 92 & & & & $\mid \begin{array}{l}4.633064225 \\
-6035\end{array}$ & \begin{tabular}{l|l}
5 & 2.5105 \\
.5007
\end{tabular} & 0.0659783333 & ${ }^{81}$ & & & & & & 0.0 .659783333 & & & $\mid \begin{array}{l}33281.96874 \\
\text { 320.1034 }\end{array}$ & ancoe \\
\hline & 139 & 879976 & & & 5.687246153 & \begin{tabular}{l|l}
3 & 6.38375 \\
\end{tabular} & 0.639216667 & 158 & 01938808 & 0.02940 & & 5.814015895 & 5.95455 & $|0.668316667|$ & 82.64888095 & 12.691095 & \begin{tabular}{|l|}
25497.19786 \\
\end{tabular} & 21253.93831 \\
\hline 3 & 106 & 560535 & 8172532 & & 9.76671085 & 6.3311 & 0.63365 & ${ }_{110}$ & 2.372466515 & 0.01725 & 0.217390695 & 9.163066957 & 7.3690666667 & 0.63365 & 82.45071429 & 8743.39081 & 37103.13262 & 21472.611226 \\
\hline 4 & 91 & 182601 & 1307 & 24 & 3.5166883321 & \begin{tabular}{l|l}
1.85536660 \\
\end{tabular} & 0.66623333 & 77 & 0.929326623 & 0.001575567 & 0.033693417 & 4.271201999 & 1.8713833333 & \begin{tabular}{|l|l|l|}
3 & 0.666233333 \\
\end{tabular} & 88.47559524 & 05.861905 & \begin{tabular}{|l|l|}
32241.21369 \\
\end{tabular} & 26189.31607 \\
\hline 5 & ${ }_{13}$ & (6488899 & 66323 & $\begin{array}{l}203942 \\
011023\end{array}$ & $\mid$ & 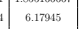 & 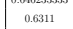 & 162 & 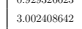 & 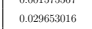 & 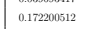 & 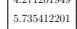 & 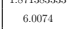 & 3 & 62286 & & & \begin{tabular}{|l}
20171.752424 \\
20171
\end{tabular} \\
\hline 6 & $9 \mathrm{~s}$ & 463605 & 730043 & 0.165239915 & 11.37652705 & \begin{tabular}{|l|l|l}
5 & 6.164233333 \\
\end{tabular} & 0.640466667 & 851.645 & 0.046537863 & 0.215726362 & 13 & \begin{tabular}{|l|l|}
6.38. \\
\end{tabular} & \begin{tabular}{|l|}
0.640466667 \\
\end{tabular} & $\begin{array}{lll}7 & 79.82471532 \\
\end{array}$ & 9862.558566 & & \begin{tabular}{|l|}
21923.39918 \\
\end{tabular} & \\
\hline 7 & & 221264 & & & 8.100386267 & \begin{tabular}{l|l|l}
7 & 5.258773333 \\
\end{tabular} & 0.642016667 & 133 & 50202677 & 0.02979202077 & 0.1 & 7.89940404 & & & 82.13552381 & & & 538.21167 \\
\hline 8 & 113 & & & & 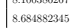 & 5 & 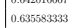 & $\begin{array}{l}1,33 \\
103 \\
\end{array}$ & s856311 & 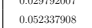 & 797 & 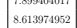 & 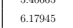 & $\left|\begin{array}{l}0.603555353333 \\
0.0353\end{array}\right|$ & 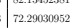 & 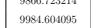 & 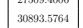 & 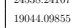 \\
\hline 9 & 9 & 18 & & & i73 & 3 & 0.6 & 77 & 566 & 0.0 & & 12.6 & 6.1616233333 & 66667 & $8=$ & & 6861 & 369 \\
\hline & 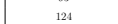 & & & & 7.215451755 & 5 6.069316667 & 0.636166667 & 140 & & 0.030 & & 6.796002416 & \begin{tabular}{|l|}
5.567776667 \\
\end{tabular} & $7 \begin{array}{l}0.636166667 \\
0.7\end{array}$ & 80.22202381 & & . & \\
\hline 11 & (2) & 3176 & & 14551077 & 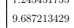 & $\mid$ & 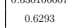 & 104 & 8912 & $\begin{array}{l}0.0437 \\
0.047 \\
0\end{array}$ & 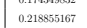 & 9.960 & 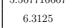 & 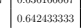 & 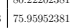 & & . & \\
\hline 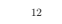 & 115 & 3913 & 0.038821737 & 77084938 & 7.7.2668s8208 & 8 & 0.062335 & ${ }_{127}$ & 8058 & 0.033371974 & 0.182679978 & 7.577470828 & $\begin{array}{ll}6.452466667 \\
\end{array}$ & 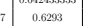 & & 3036 & 27626.90774 & 22436.45238 \\
\hline $1: 3$ & 102 & & 001 & & \begin{tabular}{|l|l|}
9.80079173 \\
\end{tabular} & & 0.632383333 & 92 & & & & & & & & & & \\
\hline 1. & 103 & & 0.035305502 & 585 & 10.81883184 & 46.5951333333 & 0.637683333 & 96 & & & & & 6.266 & \begin{tabular}{c|c|c|}
30.6376833333 \\
\end{tabular} & & & & \\
\hline 15 & 119 & .521707443 & 0.037679826 & 19412921 & \begin{tabular}{|l|l}
7.697678976 \\
\end{tabular} & \begin{tabular}{l|l|}
6 & 5.344616667
\end{tabular} & 0.6419 & 124 & 2.537029704 & 0.037312824 & .1.193165275 & 7.613835782 & 5.60305 & 0.6419 & 75.39047619 & 800.81845 & $30066.305:$ & 21295.36 \\
\hline
\end{tabular}


Table 4.5: An example of the ground truth set

\begin{tabular}{c|c|c|c|c}
\hline sequenceID & category & type & start window & end window \\
\hline \hline 0 & $\mathrm{~T}$ & HDJSTART & 0 & 0 \\
1 & $\mathrm{~W}$ & HDMAP & 1 & 136 \\
2 & $\mathrm{~T}$ & HDMAPSHUFL & 131 & 139 \\
3 & $\mathrm{~W}$ & HDSHUF & 140 & 158 \\
4 & $\mathrm{~T}$ & MIXED & 159 & 173 \\
5 & $\mathrm{~W}$ & HDSHUF & 174 & 180 \\
6 & $\mathrm{~T}$ & HDSHUFTORED & 181 & 191 \\
7 & $\mathrm{~W}$ & HDRED & 192 & 235 \\
8 & $\mathrm{~T}$ & HDREDTOREDEND & 236 & 250 \\
9 & $\mathrm{~W}$ & HDREDEND & 251 & 260 \\
10 & $\mathrm{~T}$ & HDJEND & 260 & 261 \\
\hline
\end{tabular}

\subsection{Data Preprocessing}

The workload category and workload type features have been considered as labels. These features do not participate in the clustering process but are used in the evaluation metrics.

The windows' duration could be different for each of the data files. Moreover, the scale of the reported intervals is in minutes. Consequently, Depends on the intervals, the two features, numContStarted and numContainersFinished, are normalized to one minute.

Ultimately, since the feature vectors are on different scales, the attribute with the larger amounts can take priority over others. Normalization and rescaling to the range 0 to 1 have been done using the Min-Max method:

$$
V^{\prime}=\frac{V-M i n}{M a x-M i n}
$$

\subsection{Evaluation Metrics}

Accessing the labels allowed the researchers to take advantage of the assessment methods that consider the classes' ground truth set. Given $S$ the set of actual class labels for the samples and $U$ the set of random labels assigned by a clustering 
algorithm. The metrics take into account to evaluate the five algorithms' performance on the datasets are as follows:

- Adjusted Rand Index (ARI) [26]. $a$ is defined as the number of $S$, with the same label in $U . b$ is considered the number of $S$, not with the same label in $U$.

$$
\begin{gathered}
R I=\frac{a+b}{\left(\begin{array}{c}
\left.N_{\text {samples }}\right) \\
2
\end{array}\right)} \\
A R I=\frac{R I-E(R I)}{1-E(I R)}
\end{gathered}
$$

- Fowlkes-Mallows scores (FMI) [14]. This metric calculates the geometric mean of the pairwise precision and recall. Consider $T P$ is the number of pairs of samples in the same cluster in both $S$ and $U$. FP is the number of pairs of samples in the same cluster in $S$, they do not belong to the same cluster in $U$. $F N$ is the number of pairs of samples in the same cluster in $U$, they do not belong to the same cluster in $S$.

$$
F M I=\frac{T P}{\sqrt{(T P+F P)(T P+F N)}}
$$

- Purity [2]. The class label that belongs to most of the members in a cluster is assigned as the cluster label. The purity is the percentage of the correctly assigned samples. Having one cluster for each sample ensures 100\% purity.

- Inverse Purity [2]. Unlike the purity, the inverse purity compares the cluster label, which belongs to most class members, to the cluster label for each class member. It then reports the percentage of the samples with correctly assigns labels. Having one cluster, including all data points, ensures 100\% inverse 
purity.

- V-measure [37]. This formula computes the harmonic mean of homogeneity and completeness. Homogeneity is the purity of a cluster. Completeness is the number of members with the same label assigned to the same cluster.

$$
v=2 \cdot \frac{h \cdot c}{h+c}
$$

where,

$$
\begin{aligned}
& h=1-\frac{H(S \mid U)}{H(S)} \\
& c=1-\frac{H(U \mid S)}{H(U)}
\end{aligned}
$$

To calculate the homogeneity, the classes' entropy and the conditional entropy of the classes given the cluster assignments need to be computed.

$$
\begin{gathered}
H(S)=-\sum_{s=1}^{|S|} \frac{n_{s}}{n} \cdot \log \left(\frac{n_{s}}{n}\right) \\
H(S \mid U)=-\sum_{s=1}^{|S|} \sum_{u=1}^{|U|} \frac{n_{s, u}}{n} \cdot \log ()
\end{gathered}
$$

- Workload Type accuracy (Awt). This term illustrates the percentage of actual workload types that have been found via clustering. Awt stands for Accuracy of workload type. This metric compares the clusters found by the clustering algorithm against those identified by an expert human practitioner via the ground truth files.

$$
A w t=\frac{\text { The number of Clusters }}{\text { The number of workload types in ground truth file with W lable }} \times 100
$$


- The percentage of noise points (NP).

$$
N P=\frac{\text { The number of noise workload }}{\text { Total number of workloads }} \times 100
$$

- Root Mean Square Standard Deviation (RMSSTD) [40]. This evaluation metric does not require the ground truth sets of labels. The researchers look for clusters with high homogeneity. As a result, high-performance clustering shows a low RMSSTD value. 


\section{Chapter 5}

\section{Results}

This chapter dedicates to the experimental implementation and Setup as well as performance analysis of different clustering algorithms. Moreover, it compares the results and discusses the proposed method to automate the discovery of the big data workload.

\subsection{Experimental Implementation and Setup}

The different clustering algorithms used in the experiments for this study, followed by the choice of value for their parameters, as well as the libraries used for the implementations, are described in this section. The distance metric used for all the algorithms is the Euclidean distance.

Among all evaluation metrics explained in section 4.4, the NP metric, which reports the number of noise points, is specified for the DBSCAN algorithm. The rest of the algorithms targeted in this research do not handle noise point data. Accordingly, the NP metric is solely beneficial for analyzing the DBSCAN algorithm concerning different hyperparameters to acquire the optimum eps and minPts. Since there is supposed to be no noise or outlier in the dataset, the NP metric is expected to have 
a low value. The Awt is a crucial measure in our case. To calculate the Awt, the number of clusters found via the clustering algorithm is supposed to be divided by the number of different workload types in the ground truth files. The number of identified clusters must be equal to or more than the number of workload types. Otherwise, it would mean that there are some missing workload types. We need to be able to recognize all different types of workloads and do not miss any of them. So we can ensure that any new and unseen workload type can be recognized and characterized.Finding more clusters than what is supposed to be is not harmful. In that case, by merging some of these clusters, the actual clusters are obtained. Another critical metric in this case of study is purity. Clusters should ideally contain only a single workload type. Therefore, Purity, NP, and Awt are the most critical metrics in this research. However, the researchers consider other metrics to compare different algorithms since they could give comprehensive information about the clusters' structure and exceptional situations, for instance, having high purity and Awt but low inverse purity indicates that there are more clusters than the number of workload types and that these clusters are relatively pure. As a result, the researchers do not pay attention to the low inverse purity rate.

\subsubsection{DBSCAN}

DBSCAN algorithm was applied with different values for the Eps and MinPts. DBSCAN is implemented in JAVA in this experiment. Based on the statistics of big data workloads and the Purity, Awt, and NP metrics, experiments reveal that the efficient and reasonable value for the minimum number of points in a cluster (MinPts) is 5 . To figure out the best eps parameter for the DBSCAN algorithm, the researchers employ two techniques:

1. Execute the DBSCAN for different eps value. Then, choose the best eps based 
on the Workload Type accuracy, Purity, And the percentage of noise points. The average result of implementing the DBSCAN on four different data set is provided in figure 5.1. The plots respect to each data sets are presented in the $5.2,5.3,5.4,5.5$. All experiments consider 5 as the minimum number of points (MinPts), but their radius ranges from 0.3 to 0.8 .

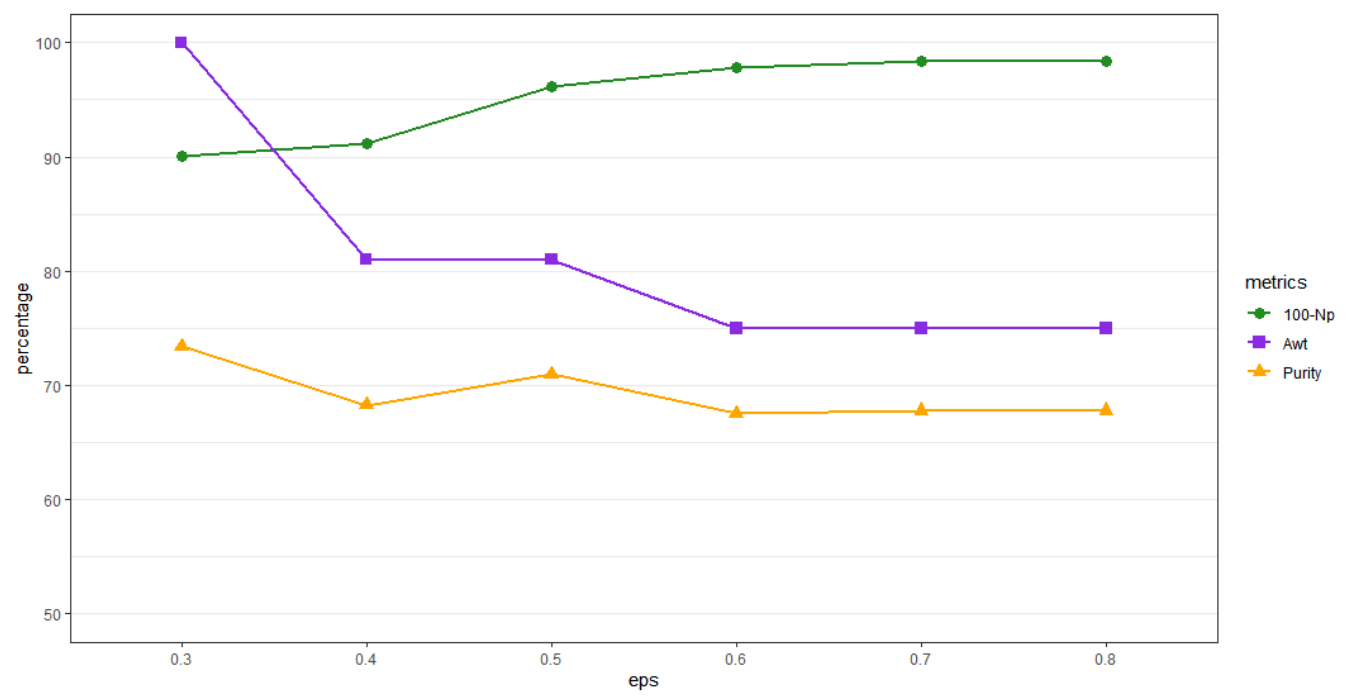

Figure 5.1: Chart for the average DBSCAN accuracy for four different data files.

2. Apply knee method to figure out the best value for eps [16]. It calculates the average distance of each point to its K-nearest neighbour. Chart respect to the average distances' sorted values reveals the optimum eps value where a significant change in the average distance values has occurred. K corresponds to the minPts value, which is 5 in this experiment. Figures 5.6, 5.7, 5.8, and 5.9 demonstrate the knee plot for each of the data files. 


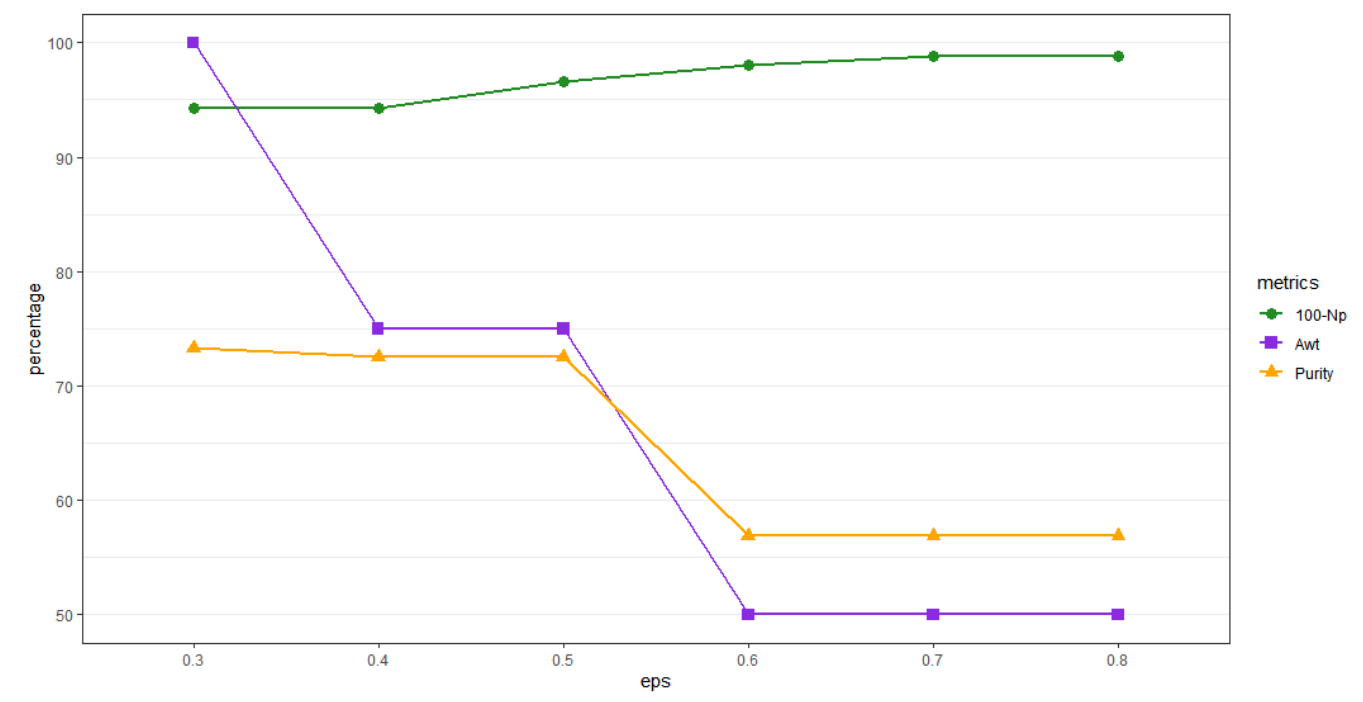

Figure 5.2: Chart for the DBSCAN accuracy for the data set Tera-Kmean. K-means starts running concurrently with Terasort when Terasort just started its reduce phase.

In conclusion, both the metrics and knee methods reveal the same results for each data file. The researchers confidently choose the optimum eps value founded by DBSCAN. DBSCAN finds 3 or 2 clusters on each data file.

\subsubsection{Optimum number of clusters}

As discussed in section 3.2.4, To detect the optimum number of clusters for clustering algorithms NbClust package [8] could be employed. The NbClust package reveals the same number of clusters as the DBSCAN algorithm for each data file.

\subsubsection{Mean Shift Clustering}

Mean shift clustering is one of the iterative algorithms. It does not require any input parameter. For each point, the algorithm analyses the field of view centred to that point. Using a mean-shift vector moves to another point toward a dense area of that field of view. The mean-shift vector is measured based on a kernel density function. 


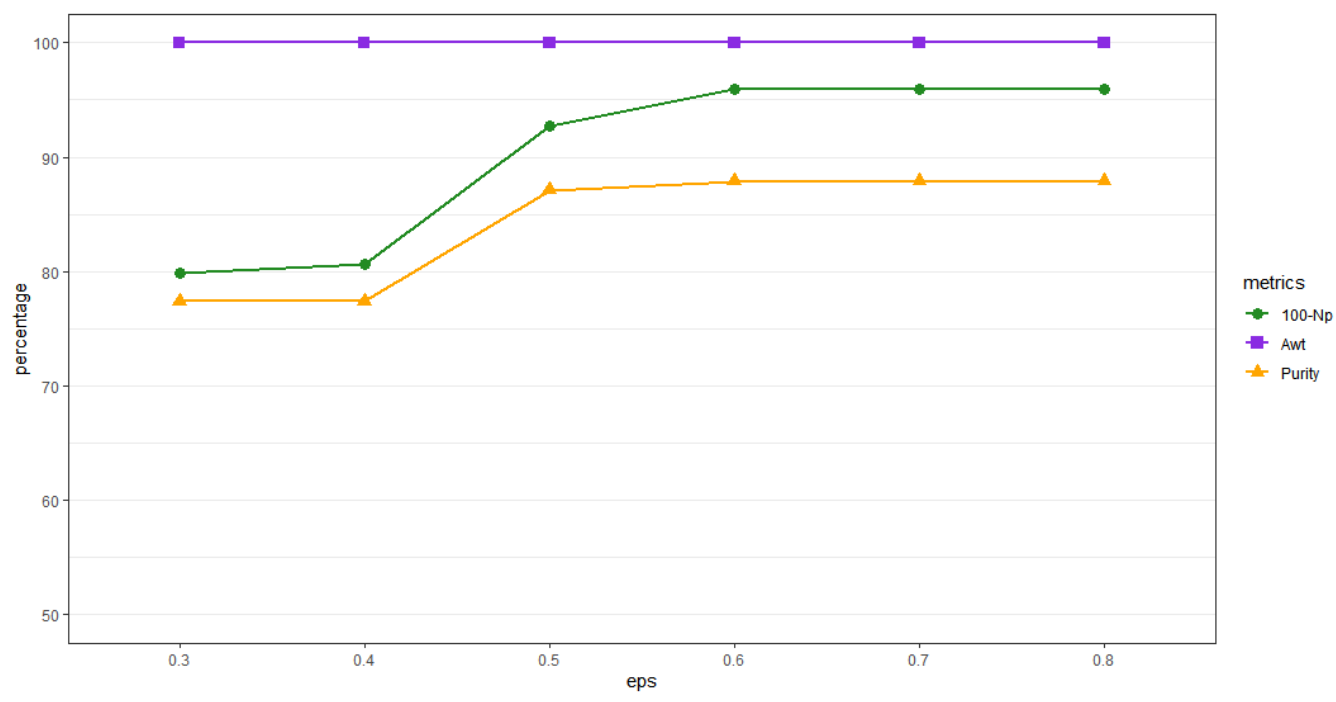

Figure 5.3: Chart for the DBSCAN accuracy for the data set Hibench-Wordcount Consequently, the field of view moves to a region that is centred on the new point. This process continues until coverage. All the points that lead to a mode are assigned to the same clusters [9]. This algorithm is implemented in python using the sklearn API.

\subsubsection{K-Means Clustering}

K-means algorithm has one input parameter, which is the number of clusters. This algorithm was run in R. It starts with 2 or 3 randomly initialized points based on each data set.

\subsubsection{Expectation Maximization (EM) clustering}

The EMCluster library is provided to implement this technique in $\mathrm{R}$. It requires the number of clusters as an input parameter, which is 2 or 3 . 


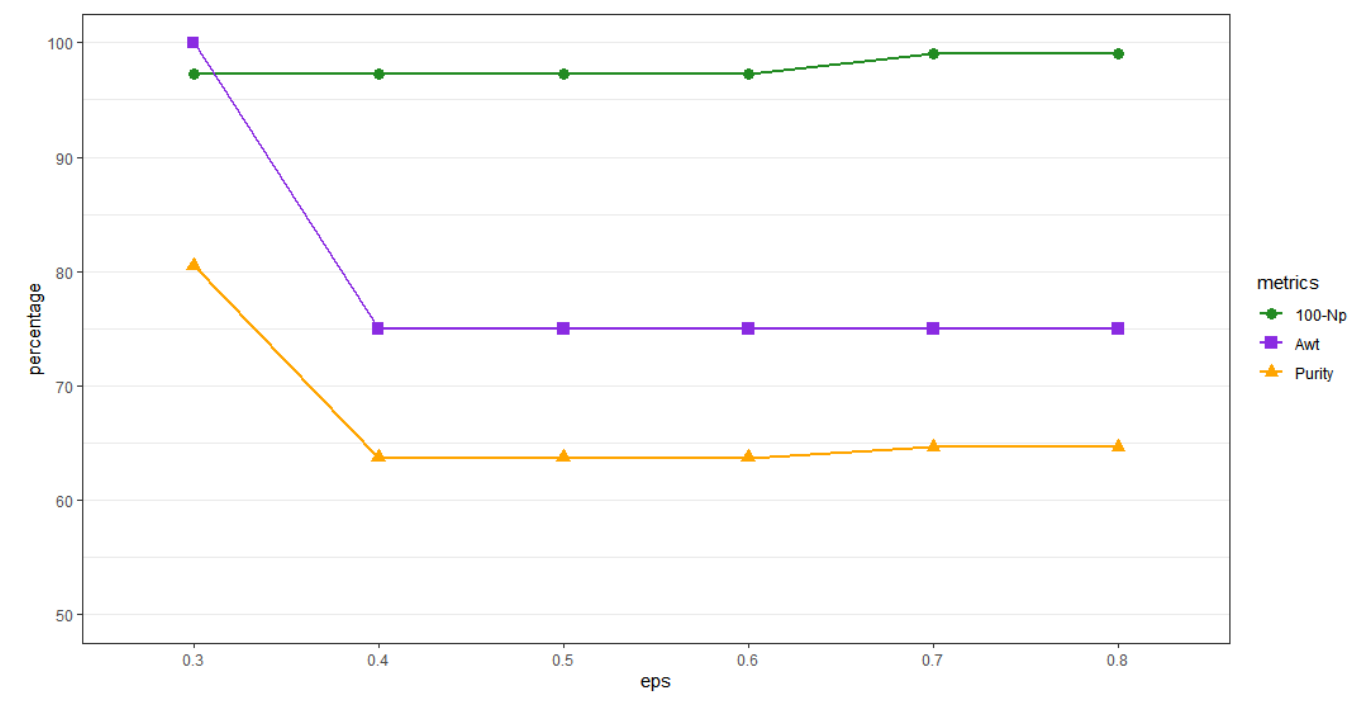

Figure 5.4: Chart for the DBSCAN accuracy for the data set Terasort

\subsubsection{Hierarchical Clustering}

The cluster package in $\mathrm{R}$ is used to apply the hierarchical clustering. Like EM clustering and K-means, 2 or 3 is the input parameter as the number of clusters. As discussed before, there are four different ways to define the distance between the clusters, including Single Linkage, Complete Linkage, Average Linkage, Centroid Linkage. The researchers considered all four distance definitions in this study. However, only the complete linkage results reveal a better result in terms of metrics are reported.

\subsection{Performance Analysis}

The average accuracy of applying K-means clustering, Simple Hierarchical clustering, DBSCAN, and EM clustering, as well as Mean-shift clustering on each of the data sets, is presented in plot 5.10. The charts associated with applying the different clustering algorithms on each of the data sets are presented in 5.11, 5.12, 5.13, 5.14. 


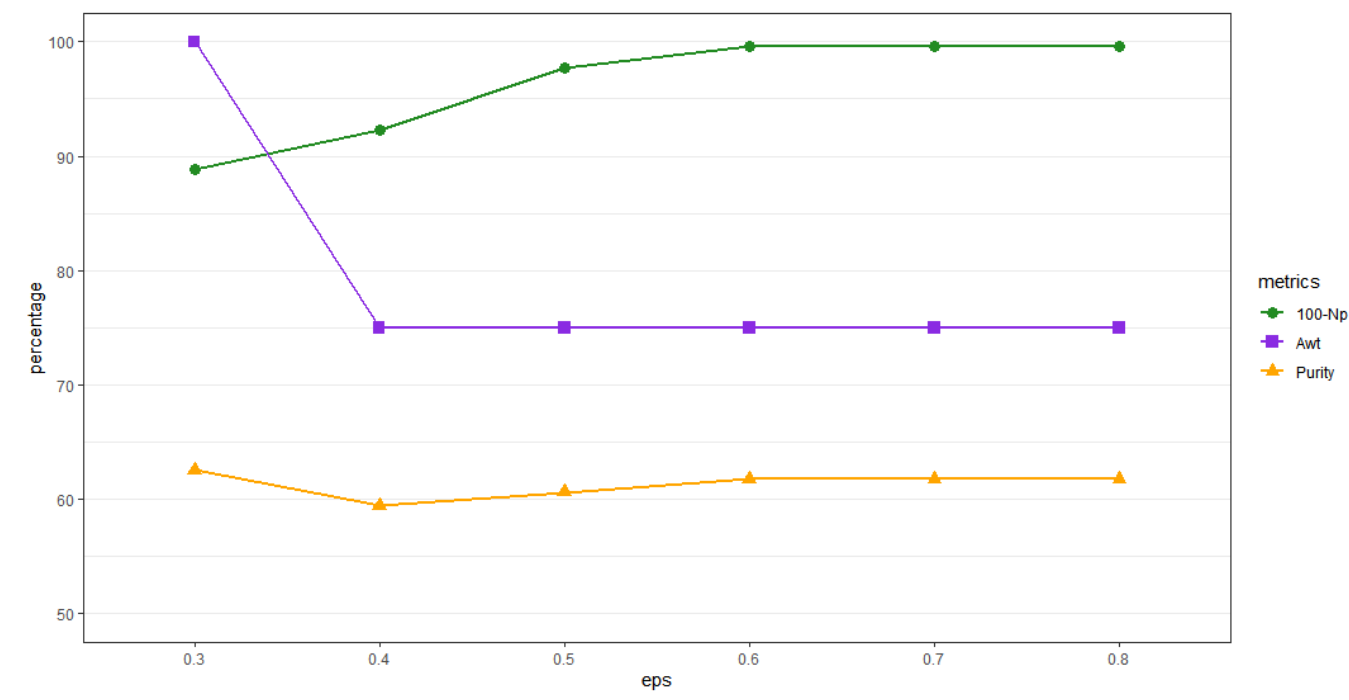

Figure 5.5: Chart for the DBSCAN accuracy for the data set Tera-K-means. Kmeans runs concurrently with Terasort.

The number of clusters is the same for all algorithms, except Mean-shift clustering. So workload type accuracy (Awt) is the same for all algorithms except Mean-shift clustering. Mean-shift clustering, which does not have any input parameter has the least value for workload type accuracy. As discussed before, the Awt is a critical measure for big data workload characterization, consequently, the researchers discarded the Mean-shift clustering.

5.10 illustrates that overall, EM clustering has the least accuracy for all kinds of measurements except purity. EM is the second least algorithm in terms of purity. As a result, EM clustering is not a possibility for the experiment as well.

As discussed earlier, the researchers were looking for clusters that are as pure as possible. They expect most of the members of a cluster to belong to the same workload type. Although the highest FMI and Inverse purity belong to the Hierarchical clustering, it demonstrates the least purity value. The cluster's distribution for the Hierarchical clustering reveals that most of the data are in one cluster, and the rest 


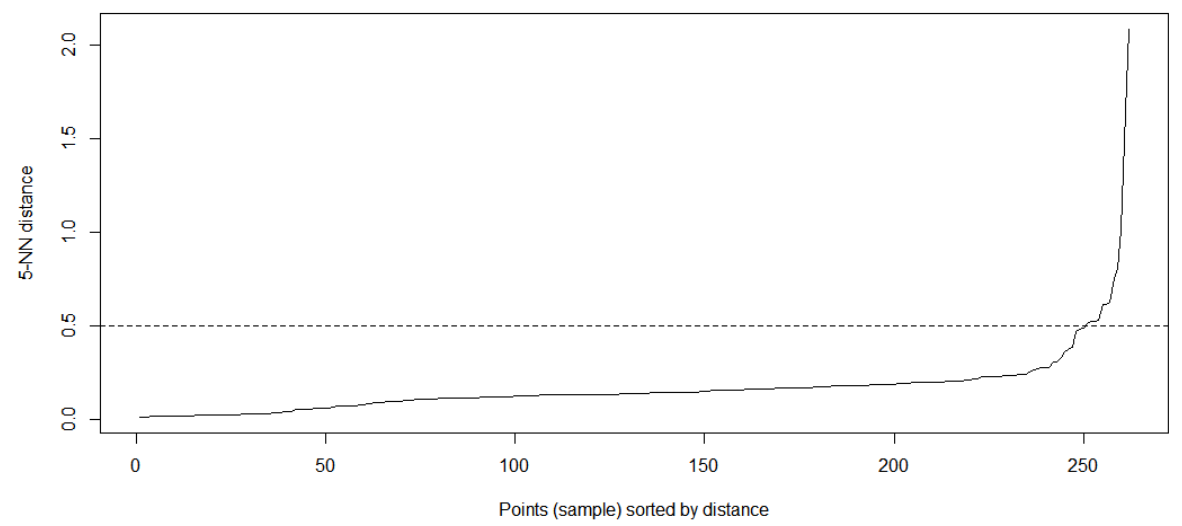

Figure 5.6: Knee plot for Tera-K-means. K-means starts running concurrently with Terasort when Terasort just started its reduce phase.

of the clusters contain one or two members. Hierarchical clustering has a high inverse purity because the inverse purity is maximized when all data are in one cluster. Since it is expected that all the clusters to have a reasonable number of members, Hierarchical clustering is not proper for this case study.

The inverse purity measures how much the members of each class have been assign to the same cluster. The purity measures how many cluster members belong to the same class. When both inverse purity and purity have low value, the clustering has very low performance, such as EM clustering. As discussed before, in cases such as Hierarchical clustering, when the inverse purity has a high value, but the purity has a low value, we conclude that most of the data have been group together in one cluster, and the rest of the clusters do not have a significant number of data. On the other hand, if there was a case where the purity had a high value, but inverse purity demonstrated low value, it would be a sign that the number of clusters is relatively high and close to the number of data points which resulted in having a few data points in all clusters. We have chosen a reasonable number of clusters based on the big data workload dataset, and the number of clusters is much less than the number 


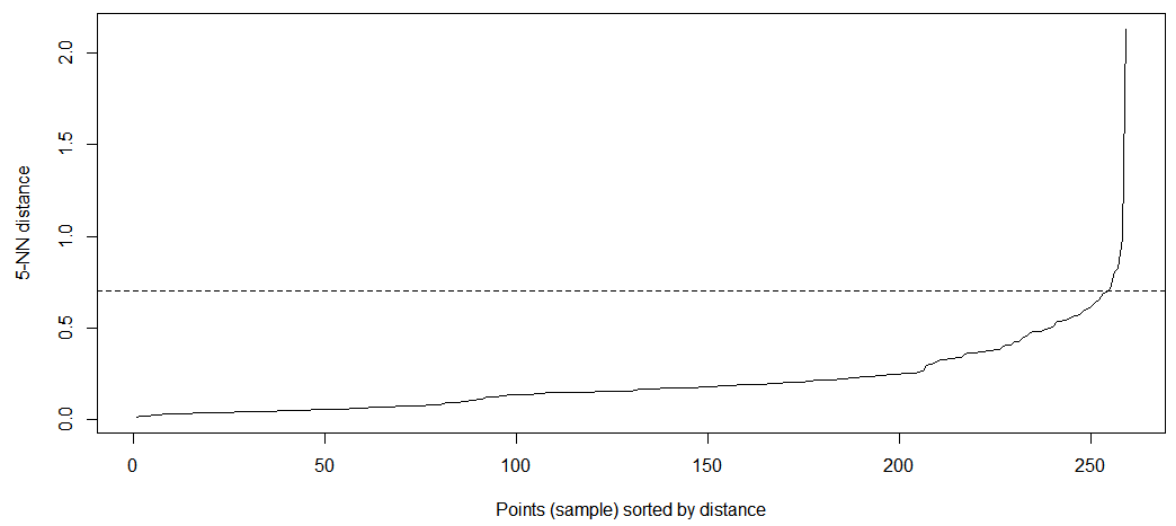

Figure 5.7: Knee plot for Tera-K-means. K-means runs concurrently with Terasort.

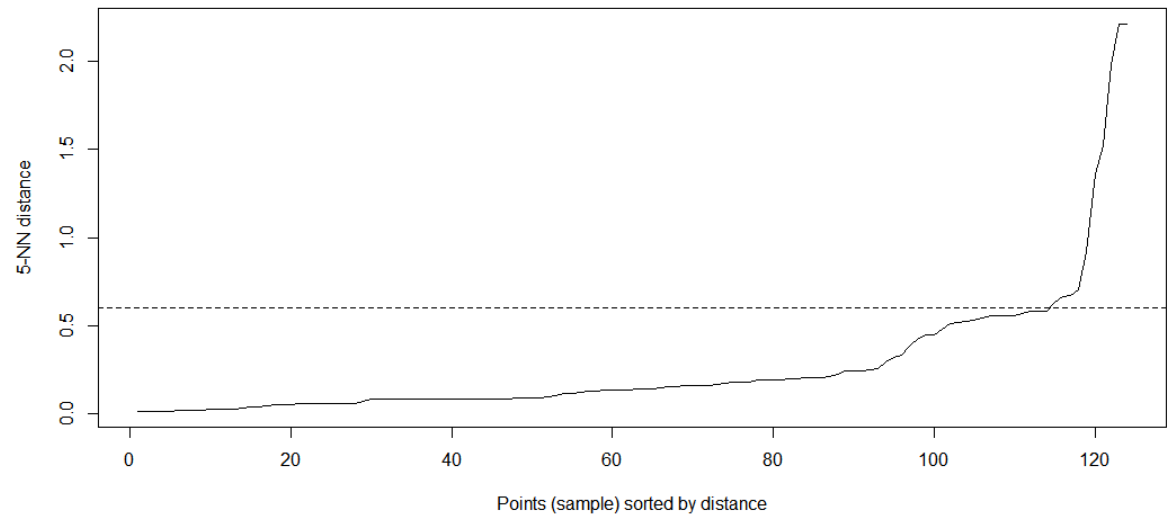

Figure 5.8: Knee plot for Hibench-Wordcount.

of data points. So, in all experiments, inverse purity has more value than purity. A high-performance clustering is one such as the K-means algorithm or DBSCAN algorithm, which reveals a high value for both the inverse purity and purity. 


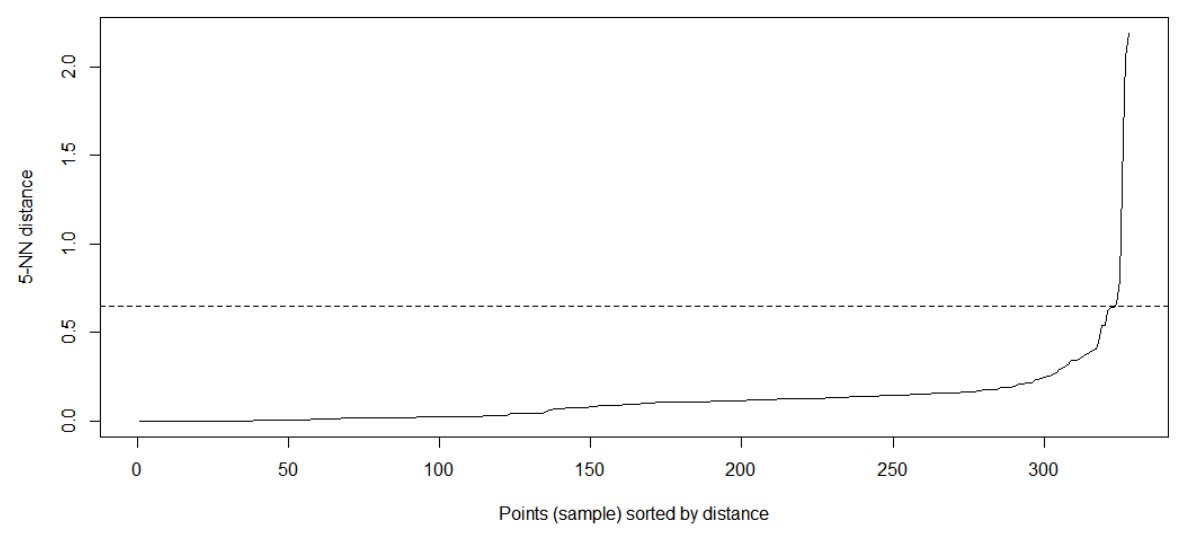

Figure 5.9: Knee plot for Terasort.

\subsection{Discussion}

Ultimately, the results of different metrics illustrate that DBSCAN and K-means techniques are the two best practical algorithms for characterizing big data workloads based on the container performance patterns. K-means performs slightly better than DBSCAN for most of the measures.

To fully automate the clustering method, it is necessary to combine the hyperparameters' automatic detection with an online version of the clustering algorithms.

To detect the optimum number of clusters for K-means, methods such as NbClust [8] could be employed. However, since online K-means [29] does not store the data points, it is not applicable to use the existing methods to determine the optimum number of clusters. Moreover, the number of big data workload types that indicate the number of clusters is unknown in advance. As a result, since the online K-means algorithm require the number of clusters as an input parameter, and it is not possible to automatically find the optimum number of clusters in the online K-means algorithm, it is not practical to use the online K-means method.

In conclusion, DBSCAN is an excellent choice to separate big data workloads 


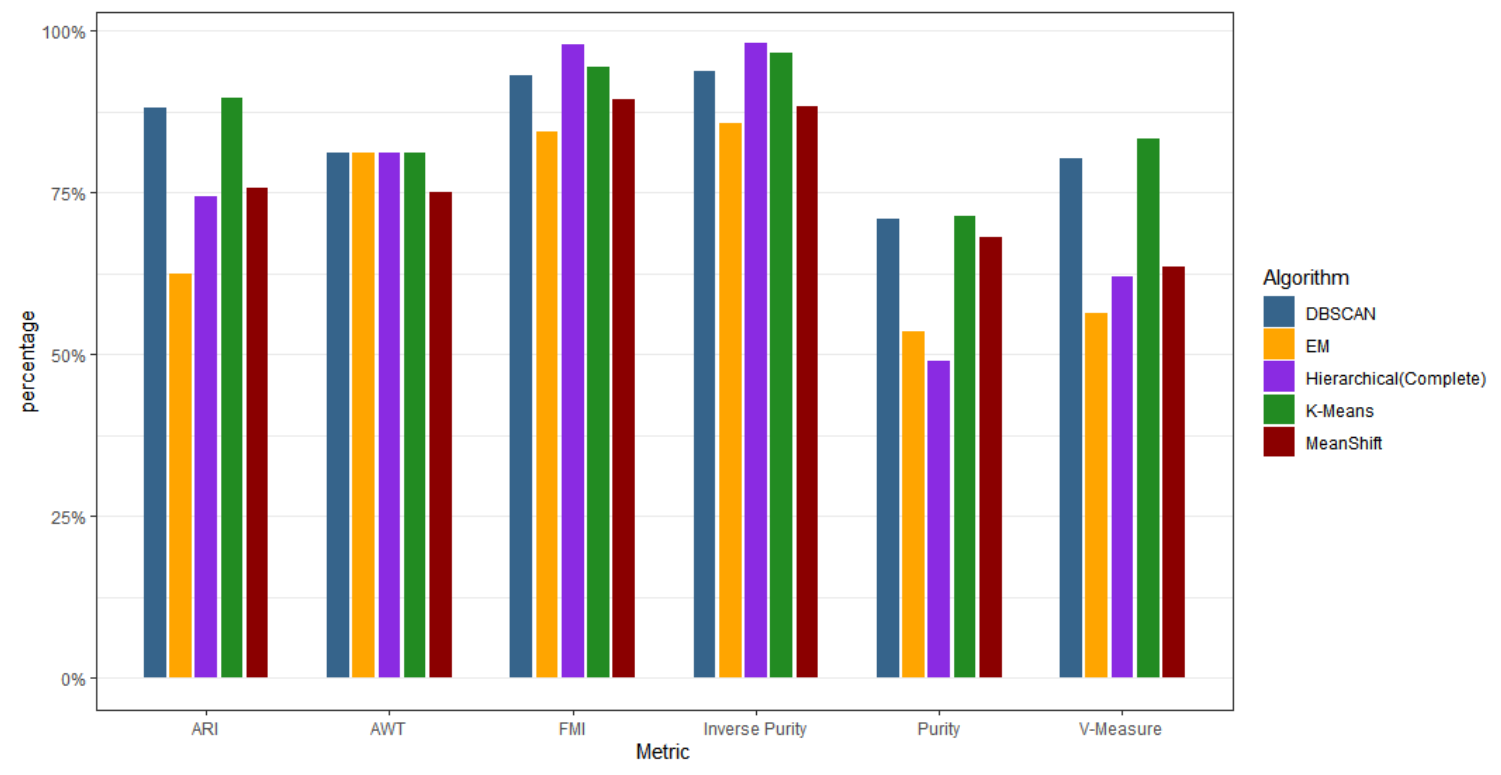

Figure 5.10: Chart for the comparison of the average accuracy in different clustering algorithms

based on their container performance features. One of the algorithms to analyze the stream data and produce the same result as DBSCAN is the Den-Stream algorithm [7].

Den-Stream requires 5 hyper-parameters: Init $N, \epsilon, \beta, \mu, \lambda$. The $\mu$ denotes the minimum number of points in a cluster, is 4 or 5 in most cases [43] and is determined based on the database. According to the researchers' knowledge and experiment on big data workloads, 5 for $\mu$ is recommended.

Den-Stream executes DBSCAN for the first InitN points. 100 to 200 could be a reasonable value for Init $N$. As the benchmarks illustrates, the experiments produced between 100 to 300 observation windows. However, it should be mentioned that real life big data applications are more complicated and more observation windows are created.

As discussed earlier, the knee-plot method employs the K-nearest neighbour distance 


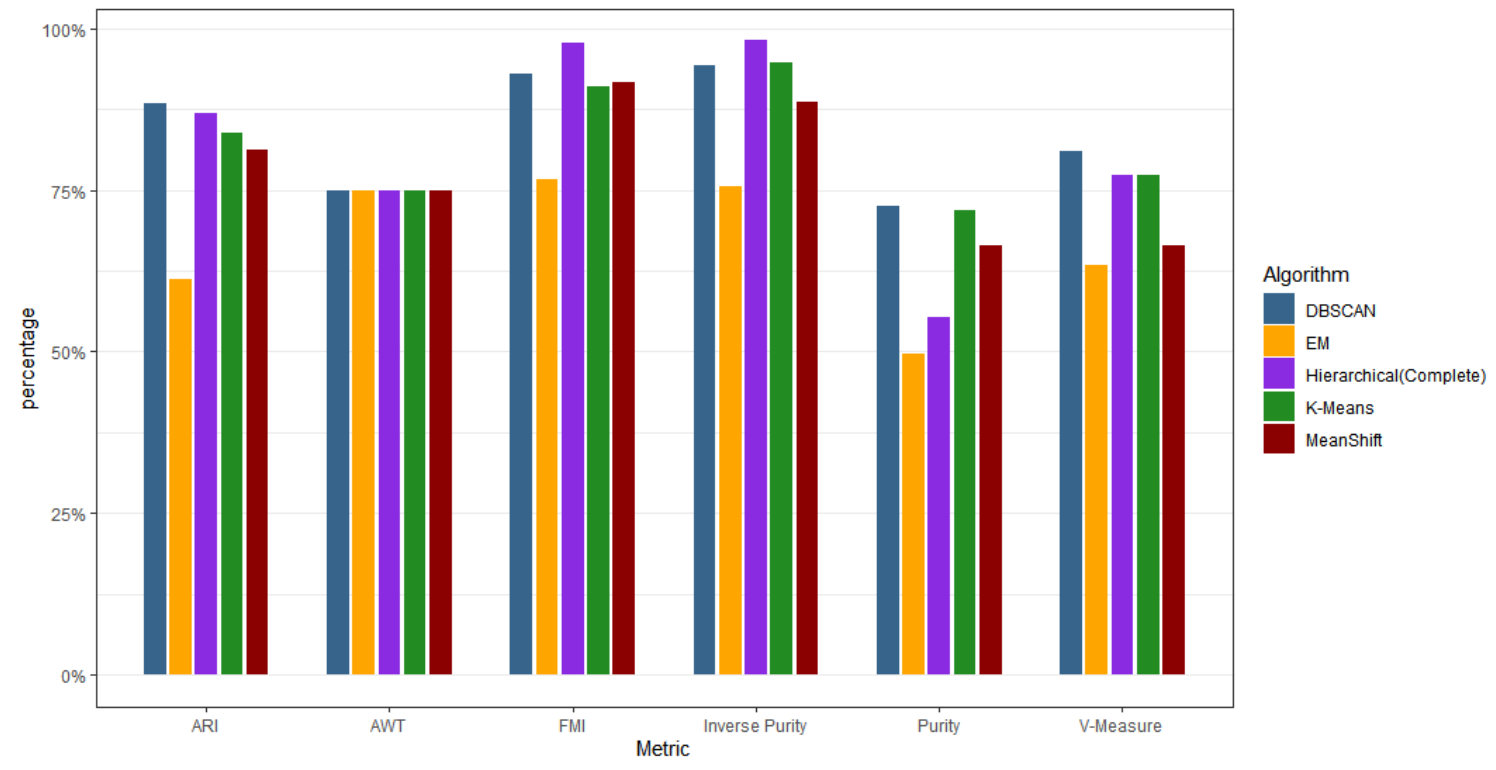

Figure 5.11: Chart for the comparison of the metrics in different clustering algorithms for the Tera-K-means workloads. K-means starts running concurrently with Terasort when Terasort just started its reduce phase.

average to find the optimum $\epsilon$ for the DBSCAN algorithm. Satopaa et al. developed the Kneedle algorithm to detect the knee point in a plot [39]. In this study the researchers use the KneeLocator function provided in kneed package [4] in python to detect the knee point in a plot automatically. The knee strategy could be used, and the critical point corresponds to the $\epsilon$ can be determined automatically using a function such as kneeLocator [4]. Then, this $\epsilon$ is passed to the DBSCAN to find the initial p-micro-clusters. After setting the $\mu$ and $\epsilon$, to find the optimum decay factor $\lambda$, the $\operatorname{Init} N$ is set to $\operatorname{Init} N / 2$. By using $\operatorname{Init} N$ number of points to find the $\lambda$ and $\beta$, the Den-Stream algorithm is executed for different $0<\lambda<1$ values while $\beta$ has a constant value such as 0.5 . Then the root mean square standard deviation (RMSSTD) of the clustering is calculated. Homogeneity and RMSSTD have an inverse relationship. The optimum $\lambda$ is where the RMSSTD is minimum. Finally, to figure out the optimum value for outliers threshold $0<\beta<1$, the same method is employed to get the minimum RMSSTD. 


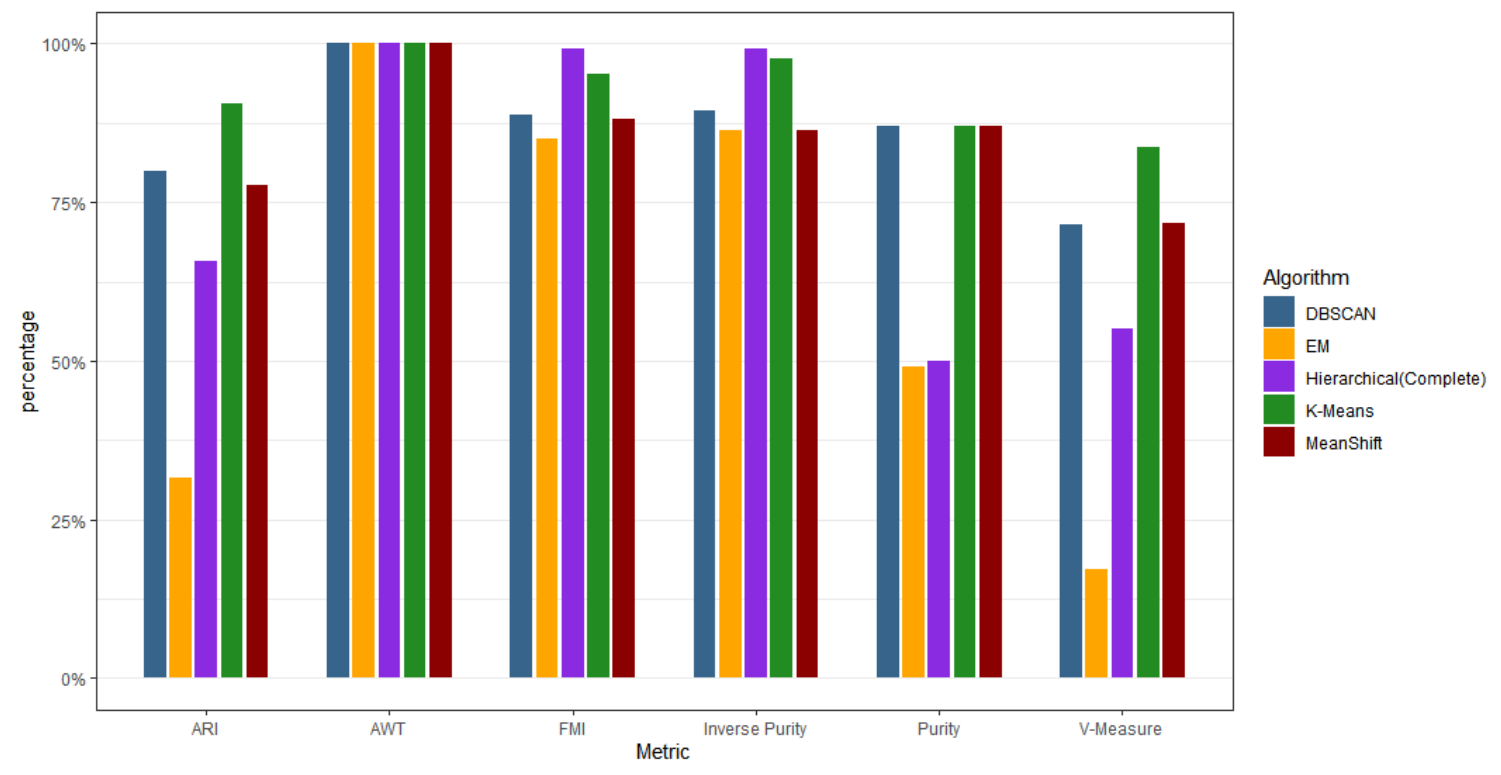

Figure 5.12: Chart for the comparison of the metrics in different clustering algorithms for the Hibench-Wordcount workloads

Owing to the number of observation windows that do not represent a massive number of data points, it is possible to take advantage of the incremental DBSCAN algorithm [11] which preserves all the data points. Same as DBSCAN, this algorithm only needs the $\epsilon$, and $\mu$ as input parameters. Concerning five as the minimum number of points, $\epsilon$ is obtained by employing the Knee method on the Init $N$ initial data points. DBSCAN is applied to the Init $N$ initial observation windows. Then, each new observation window is characterized one by one using the incremental DBSCAN algorithm.

In conclusion, a batch phase of the Den-Streams and Incremental DBSCAN enabled us to find the required hyper-parameters. Both of these prototypes can be used to characterize the observation windows online after characterizing the first InitN points in batch. Based on the memory capacity as well as the size of the observation windows dataset, the proper technique can be chosen.

The accuracy of clustering techniques in characterizing big data workloads is 


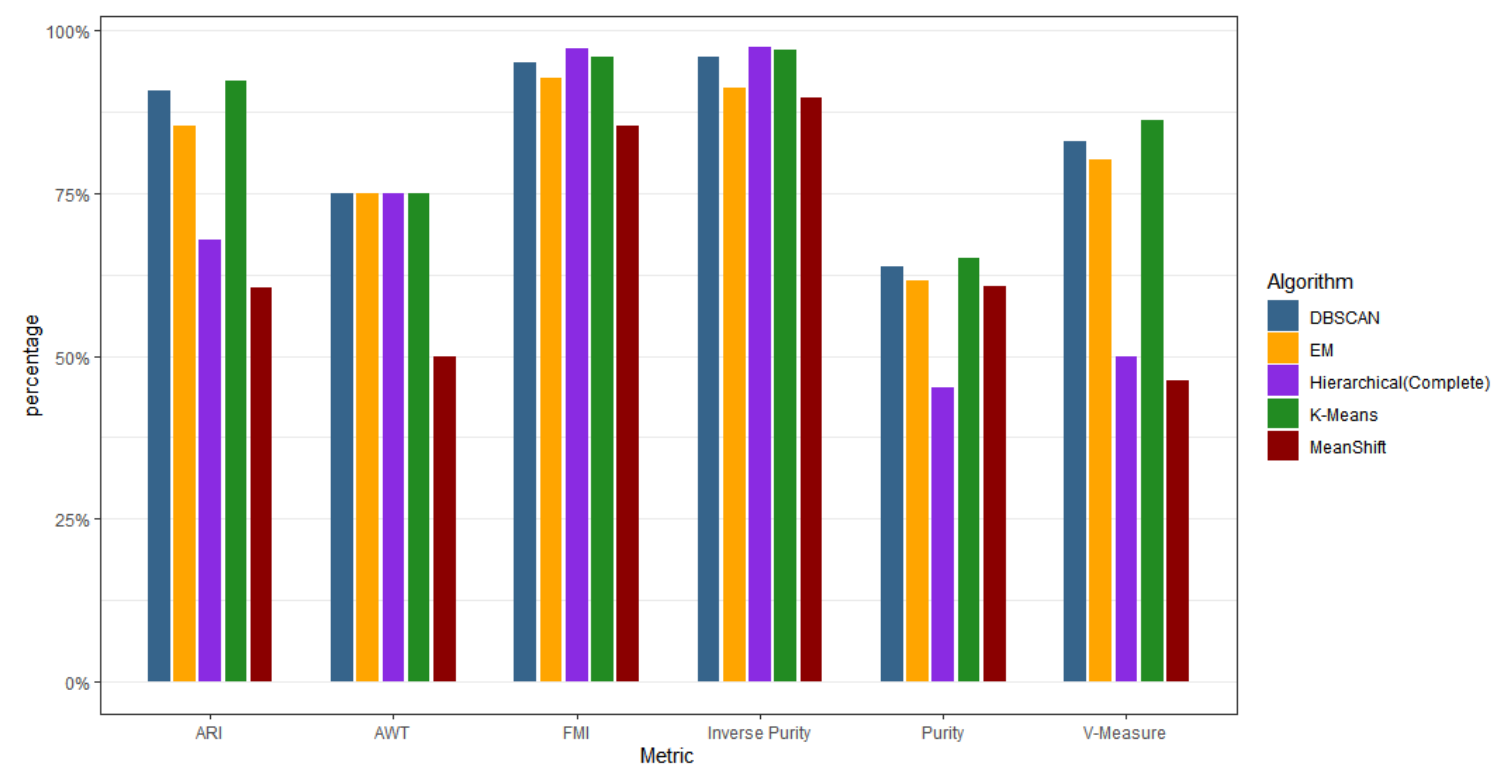

Figure 5.13: Chart for the comparison of the metrics in different clustering algorithms for the Terasort workloads

slightly less than humans labelling the data. However, by using the automated discovery of big data workloads, we can recognize unseen and new workloads, which is impossible to have a human label the dataset regularly. As a result, automatically characterizing the big data workloads outperforms characterizing by humans and improves the performance of the big data workload prediction presented by Genkin and Dehne [17]. 


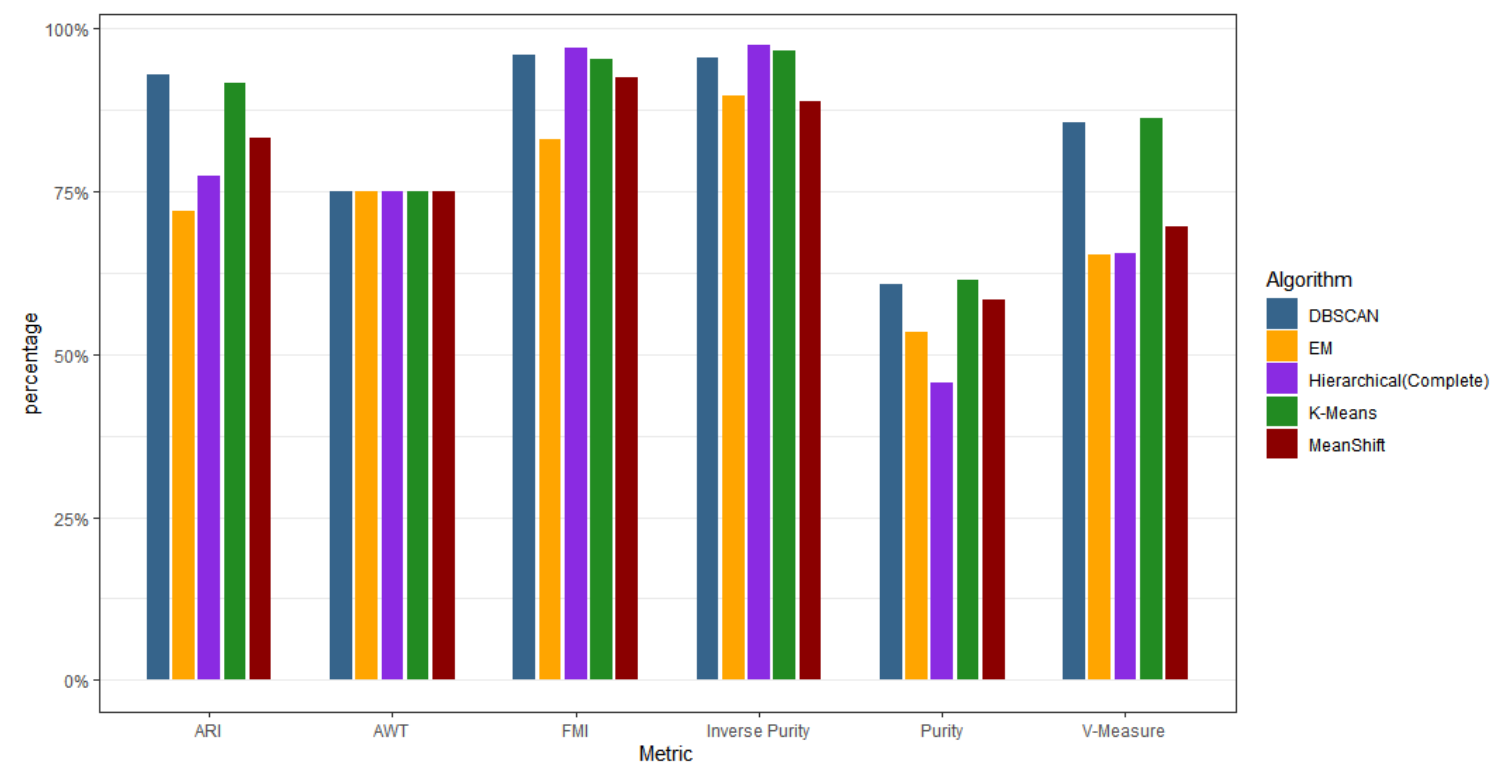

Figure 5.14: Chart for the comparison of the metrics in different clustering algorithms for the Tera-K-means workloads. K-means runs concurrently with Terasort. 


\section{Chapter 6}

\section{Conclusions and Future Work}

This work aims to reduce the amount of human intervention needed to identify different workload types. Supervised machine learning algorithms can not be used to recognize previously unseen, and consequently un-labelled workloads. The DBSCAN algorithm was observed to achieve the best results. The key finding of the thesis is that clustering finds, with a high degree of accuracy, the same workloads as those identified by an expert human practitioner.

Workload classification algorithms deal with streams of big data workloads. To identify and separate these workloads, we apply the Den-Stream and Incremental DBSCAN techniques along with the methods to automatically identify the system parameters. Although both of the proposed methods still depend on initial historical data and are not fully automated from the beginning, the proposed method is capable of detecting new workloads. The current categories will be detected based on the incoming data and could change during time. Using clustering techniques, different workload types can be automatically discovered. Then, it is possible to automate the labelling phase for downstream processing by supervised learning classifiers utilized in big data workload prediction algorithms.

This research focuses on the Hadoop and Spark workloads and analyzes the data 
related to these frameworks. However, the proposed method to identify different workloads can be generalized for other analytic applications.

Potential further studies could be to characterize the workloads starts from the first input data and not wait to receive a sufficient amount of data, to begin. Moreover, removing the initial phase requires finding a technique to update the hyperparameters during the clustering process. Furthermore, other online and streaming algorithms related to the stream and online version DBSCAN algorithm could be analyzed in terms of the possibility of making workload discovery completely online. 


\section{List of References}

[1] What is the coefficient of variation? https://www.statisticshowto.com/ probability-and-statistics/how-to-find-a-coefficient-of-variation, 2020 .

[2] Enrique Amigó, Julio Gonzalo, Javier Artiles, and Felisa Verdejo. A comparison of extrinsic clustering evaluation metrics based on formal constraints. Information retrieval, 12(4):461-486, 2009.

[3] Akhil Arora and Shrey Mehrotra. Learning Yarn. Packt Publishing Ltd, 2015.

[4] Kevin Arvai. Knee/elbow point detection. https://www.kaggle.com/ kevinarvai/knee-elbow-point-detection, December 2019.

[5] Ahsan Javed Awan, Mats Brorsson, Vladimir Vlassov, and Eduard Ayguade. Micro-architectural characterization of apache spark on batch and stream processing workloads. In 2016 IEEE International Conferences on Big Data and Cloud Computing (BDCloud), Social Computing and Networking (SocialCom), Sustainable Computing and Communications (SustainCom)(BDCloudSocialCom-SustainCom), pages 59-66. IEEE, 2016.

[6] Craig Borysowich. Better kiviat diagrams. https://www.toolbox.com/ tech/enterprise-software/blogs/better-kiviat-diagrams-101907, October 2007 .

[7] Feng Cao, Martin Estert, Weining Qian, and Aoying Zhou. Density-based clustering over an evolving data stream with noise. In Proceedings of the 2006 SIAM international conference on data mining, pages 328-339. SIAM, 2006.

[8] Malika Charrad, Nadia Ghazzali, Véronique Boiteau, Azam Niknafs, and Maintainer Malika Charrad. Package 'nbclust'. Journal of statistical software, 61:1-36, 2014. 
[9] Dorin Comaniciu and Peter Meer. Mean shift: A robust approach toward feature space analysis. IEEE Transactions on pattern analysis and machine intelligence, 24(5):603-619, 2002.

[10] Jeffrey Dean and Sanjay Ghemawat. Mapreduce: simplified data processing on large clusters. Communications of the ACM, 51(1):107-113, 2008.

[11] Martin Ester, H. Kriegel, J. Sander, M. Wimmer, and Xiaowei Xu. Incremental clustering for mining in a data warehousing environment. In $V L D B, 1998$.

[12] Martin Ester, Hans-Peter Kriegel, Jörg Sander, Xiaowei Xu, et al. A densitybased algorithm for discovering clusters in large spatial databases with noise. In $K d d$, volume 96, pages 226-231, 1996.

[13] The Apache Software Foundation. Apache hadoop yarn. https://hadoop. apache.org/docs/current/hadoop-yarn/hadoop-yarn-site/YARN . html, July 2020.

[14] Edward B Fowlkes and Colin L Mallows. A method for comparing two hierarchical clusterings. Journal of the American statistical association, 78(383):553-569, 1983.

[15] Jerome H Friedman. Stochastic gradient boosting. Computational statistics \& data analysis, 38(4):367-378, 2002.

[16] Manisha Naik Gaonkar and Kedar Sawant. Autoepsdbscan: Dbscan with eps automatic for large dataset. International Journal on Advanced Computer Theory and Engineering, 2(2):11-16, 2013.

[17] Mikhail Genkin and Frank Dehne. Autonomic workload change classification and prediction for big data workloads. In 2019 IEEE International Conference on Big Data (Big Data), pages 2835-2844. IEEE, 2019.

[18] Mikhail Genkin, Frank Dehne, Pablo Navarro, and Siyu Zhou. Machine-learning based spark and hadoop workload classification using container performance patterns. In International Symposium on Benchmarking, Measuring and Optimization, pages 118-130. Springer, 2018.

[19] Mikhail Genkin, Frank Dehne, Maria Pospelova, Yabing Chen, and Pablo Navarro. Automatic, on-line tuning of yarn container memory and cpu parameters. In 2016 IEEE 18th International Conference on High Performance Computing and Communications; IEEE 14th International Conference 
on Smart City; IEEE 2nd International Conference on Data Science and Systems (HPCC/SmartCity/DSS), pages 317-324. IEEE, 2016.

[20] Zoubin Ghahramani. Unsupervised learning. In Summer School on Machine Learning, pages 72-112. Springer, 2003.

[21] Stephanie Glen. Welch's test for unequal variances. https://statisticshowto. datasciencecentral.com/welchs-test-for-unequal-variances, May 2019.

[22] Google. Google cluster data v1. http://code.google.com/p/ googleclusterdata/wiki/TraceVersion1.

[23] Joseph L. Hellerstein. Google cluster data. http://googleresearch . blogspot. com/2010/01/google-cluster-data.html, January 2010.

[24] Sepp Hochreiter and Jürgen Schmidhuber. Long short-term memory. Neural computation, 9(8):1735-1780, 1997.

[25] Shengsheng Huang, Jie Huang, Yan Liu, Lan Yi, and Jinquan Dai. Hibench: A representative and comprehensive hadoop benchmark suite. In Proc. ICDE Workshops, pages 41-51, 2010.

[26] Lawrence Hubert and Phipps Arabie. Comparing partitions. Journal of classification, 2(1):193-218, 1985.

[27] Zhen Jia, Jianfeng Zhan, Lei Wang, Rui Han, Sally A McKee, Qiang Yang, Chunjie Luo, and Jingwei Li. Characterizing and subsetting big data workloads. In 2014 IEEE International Symposium on Workload Characterization (IISWC), pages 191-201. IEEE, 2014.

[28] Ian T Jolliffe and Jorge Cadima. Principal component analysis: a review and recent developments. Philosophical Transactions of the Royal Society A: Mathematical, Physical and Engineering Sciences, 374(2065):20150202, 2016.

[29] Angie King. Online k-means clustering of nonstationary data. Prediction Project Report, pages 1-9, 2012.

[30] Weiwei Lin, Ziming Wu, Longxin Lin, Angzhan Wen, and Jin Li. An ensemble random forest algorithm for insurance big data analysis. Ieee access, 5:16568$16575,2017$. 
[31] Ruirui Lu, Gang Wu, Bin Xie, and Jingtong Hu. Stream bench: Towards benchmarking modern distributed stream computing frameworks. In 2014 IEEE/ACM 7th International Conference on Utility and Cloud Computing, pages 69-78. IEEE, 2014.

[32] Chunjie Luo, Jianfeng Zhan, Zhen Jia, Lei Wang, Gang Lu, Lixin Zhang, ChengZhong Xu, and Ninghui Sun. Cloudrank-d: benchmarking and ranking cloud computing systems for data processing applications. Frontiers of Computer Science, 6(4):347-362, 2012.

[33] Asit K Mishra, Joseph L Hellerstein, Walfredo Cirne, and Chita R Das. Towards characterizing cloud backend workloads: insights from google compute clusters. ACM SIGMETRICS Performance Evaluation Review, 37(4):34-41, 2010.

[34] Ismael Solis Moreno, Peter Garraghan, Paul Townend, and Jie Xu. An approach for characterizing workloads in google cloud to derive realistic resource utilization models. In 2013 IEEE Seventh International Symposium on Service-Oriented System Engineering, pages 49-60. IEEE, 2013.

[35] Owen O'Malley. Terabyte sort on apache hadoop. Yahoo, available online at: http://sortbenchmark. org/Yahoo-Hadoop. pdf,(May), pages 1-3, 2008.

[36] Duc Truong Pham, Stefan S Dimov, and Chi D Nguyen. Selection of k in kmeans clustering. Proceedings of the Institution of Mechanical Engineers, Part C: Journal of Mechanical Engineering Science, 219(1):103-119, 2005.

[37] Andrew Rosenberg and Julia Hirschberg. V-measure: A conditional entropybased external cluster evaluation measure. In Proceedings of the 2007 joint conference on empirical methods in natural language processing and computational natural language learning (EMNLP-CoNLL), pages 410-420, 2007.

[38] T Sajana, CM Sheela Rani, and KV Narayana. A survey on clustering techniques for big data mining. Indian journal of Science and Technology, 9(3):1-12, 2016.

[39] Ville Satopaa, Jeannie Albrecht, David Irwin, and Barath Raghavan. Finding a" kneedle" in a haystack: Detecting knee points in system behavior. In 2011 31st international conference on distributed computing systems workshops, pages 166-171. IEEE, 2011.

[40] Sughash Sharma. Applied multivariate techniques. 1996. 
[41] Kyuseok Shim. Mapreduce algorithms for big data analysis. Proceedings of the VLDB Endowment, 5(12):2016-2017, 2012.

[42] Apache Spark. Apache spark. Retrieved January, 17:2018, 2018.

[43] Artur Starczewski, Piotr Goetzen, and Meng Joo Er. A new method for automatic determining of the dbscan parameters. Journal of Artificial Intelligence and Soft Computing Research, 10(3):209-221, 2020.

[44] Lei Wang, Jianfeng Zhan, Chunjie Luo, Yuqing Zhu, Qiang Yang, Yongqiang He, Wanling Gao, Zhen Jia, Yingjie Shi, Shujie Zhang, et al. Bigdatabench: A big data benchmark suite from internet services. In 2014 IEEE 20th international symposium on high performance computer architecture (HPCA), pages 488-499. IEEE, 2014.

[45] Tom White. Hadoop: The definitive guide. " O'Reilly Media, Inc.", 2012.

[46] Wen Xiong, Zhibin Yu, Zhendong Bei, Juanjuan Zhao, Fan Zhang, Yubin Zou, Xue Bai, Ye Li, and Chengzhong Xu. A characterization of big data benchmarks. In 2013 IEEE international conference on big data, pages 118-125. IEEE, 2013.

[47] Wen Xiong, Zhibin Yu, Lieven Eeckhout, Zhengdong Bei, Fan Zhang, and Chengzhong Xu. Szts: A novel big data transportation system benchmark suite. In 2015 44th International Conference on Parallel Processing, pages 819-828. IEEE, 2015.

[48] Rui Xu and Donald Wunsch. Survey of clustering algorithms. IEEE Transactions on neural networks, 16(3):645-678, 2005.

[49] Miin-Shen Yang, Chien-Yo Lai, and Chih-Ying Lin. A robust em clustering algorithm for gaussian mixture models. Pattern Recognition, 45(11):3950-3961, 2012.

[50] Ahmad Yasin. A top-down method for performance analysis and counters architecture. In 2014 IEEE International Symposium on Performance Analysis of Systems and Software (ISPASS), pages 35-44. IEEE, 2014.

[51] Zhibin Yu, Wen Xiong, Lieven Eeckhout, Zhendong Bei, Avi Mendelson, and Chengzhong Xu. Mia: Metric importance analysis for big data workload characterization. IEEE Transactions on Parallel and Distributed Systems, 29(6):1371$1384,2017$. 
[52] Matei Zaharia, Mosharaf Chowdhury, Michael J Franklin, Scott Shenker, Ion Stoica, et al. Spark: Cluster computing with working sets. HotCloud, 10(1010):95, 2010. 